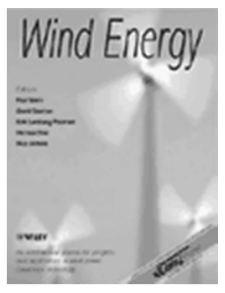

\title{
Testing of self-similarity and helical symmetry in vortex generator flow simulations
}

\begin{tabular}{|r|l|}
\hline Journal: & Wind Energy \\
\hline Manuscript ID: & WE-14-0055.R2 \\
\hline Wiley - Manuscript type: & Research Article \\
\hline Date Submitted by the Author: & n/a \\
\hline Complete List of Authors: & $\begin{array}{l}\text { FERNANDEZ-GAMIZ, UNAI; University of the Basque Country, } \\
\text { Velte, Clara Marika; Technical University of Denmark, Department of } \\
\text { Mechanical Engineering } \\
\text { Réthoré, Pierre-Elouan; Technical University of Denmark, Department of } \\
\text { Wind Energy } \\
\text { Sørensen, Niels; Technical University of Denmark, Department of Wind } \\
\text { Energy } \\
\text { Egusquiza, Eduard; Technical University of Catalunya, Fluid Mechanics } \\
\text { Department }\end{array}$ \\
\hline Keywords: & $\begin{array}{l}\text { Vortex generator, wake, computational fluid dynamics, aerodynamics, wind } \\
\text { turbine }\end{array}$ \\
\hline
\end{tabular}




\title{
RESEARCH ARTICLE
}

\section{Testing of self-similarity and helical symmetry in vortex generator flow simulations}

\author{
U. Fernández-Gámiz ${ }^{1}$, C. M. Velte ${ }^{2}$, P.-E. Réthoré ${ }^{3}$, N. N. Sørensen ${ }^{3}$ and E. Egusquiza ${ }^{4}$ \\ ${ }^{1}$ Nuclear Engineering and Fluid Mechanics Department, University of the Basque Country, Nieves Cano 12, 01006 Vitoria-Gasteiz, \\ Araba, Spain. \\ ${ }^{2}$ Section of Fluid Mechanics, Coastal and Maritime Engineering, Department of Mechanical Engineering, Technical University of \\ Denmark, Nils Koppels Allé, 2800 Kgs. Lyngby, Denmark. \\ ${ }^{3}$ DTU Wind Energy Department, Technical University of Denmark, Risø Campus, Bldg 115, Frederiksborgvej 399, DK-4000 \\ Roskilde, Denmark. \\ ${ }^{4}$ Technical University of Catalonia, Fluid Mechanics Department, Av. Diagonal, 64708028 Barcelona Spain.
}

\begin{abstract}
Vortex generators (VG) are used increasingly by wind turbine industry as flow control devices to improve rotor blade performance. According to experimental observations, the vortices generated by VGs have previously been observed to be self-similar for both the axial $\left(u_{z}\right)$ and azimuthal $\left(u_{\theta}\right)$ velocity components. Furthermore, the measured vortices have been observed to obey the criteria for helical symmetry. These are powerful results, since it reduces the highly complex 3D-flow to merely four parameters and therefore significantly facilitates the modeling of this type of flow, which in a larger perspective can assist in parametric studies to increase the total power output of wind turbines. In this study, corresponding computer simulations using Reynolds-Averaged Navier-Stokes equations have been carried out and compared to the experimental observations. The main objective is to investigate how well the simulations can reproduce these aspects of the physics of the flow, i.e., investigate if the same analytical model can be applied and therefore significantly facilitates the modeling of this type of flow, which in a larger perspective can assist in parametric studies to increase the total power output of wind turbines. This is especially interesting since these types of flows are notoriously difficult for the turbulence models to predict correctly. Using this model, parametric studies can be significantly reduced and, moreover, reliable simulations can substantially reduce the costs of the parametric studies themselves. Copyright (c) 2010 John Wiley \& Sons, Ltd.
\end{abstract}

\section{KEYWORDS}

Vortex generator; wake; CFD; computational fluid dynamics; aerodynamics; wind turbine.

\section{Correspondence}

U. Fernández-Gámiz, Nuclear Engineering and Fluid Mechanics Department, University of the Basque Country, Nieves Cano 12, 01006 Vitoria-Gasteiz, Araba, Spain.

E-mail: unai.fernandez@ehu.es

Received... 


\section{INTRODUCTION}

Research has been carried out on Vortex Generators (VGs) in applied aerodynamics on airplane wings for more than sixty years $[1,2,3]$. VGs are passive devices for flow control that enhance mixing of the boundary layer and can thus transfer high momentum fluid closer to the wall and thereby suppress separation [4]. An extensive range of conventional vortex generators are in use for successful engineering applications of flow separation control, since controlled near-wall vortices can transport both heat and momentum, aiding in cooling and re-energizing the lowest part of the boundary layer. The fluid particles with great streamwise momentum are swept along helical trajectories toward the surface to mix with and, to some extend, to restore the retarded near-wall flow. Therefore, it is important to be able to control and optimize parameters such as the vortex strength and vortex core size to the existing flow setting, [5]. On wind turbine blades VGs are usually mounted in a spanwise array on the suction side of the blade and have the advantage that they can be added as a post-production fix to blades that do not perform as expected. An overview of different airfoils with several VG options is listed in van Rooij et al. [6]. They are further frequently employed on, e.g., wind turbine blades to enable production of more slender blades. That is, the width of a blade can be reduced, thereby also reducing the weight for the same load distribution and power production and thus facilitating up-scaling of the turbines.

These devices are commonly triangular or rectangular vanes inclined at an angle of attack to the oncoming flow. They are normally dimensioned in relation to the local boundary layer thickness to obtain optimal interaction between the vortex and boundary layer, and are frequently arranged in pairs, producing counter-rotating vortices, upstream of the flow separation area [7, 8]. Basic research on Vortex Generators mounted on a flat plate has previously been the concern of several researchers, see e.g. Lin [7]. For an example of successful control of a laminar separation bubble with significant drag reduction, see the research at moderate Reynolds number carried out by Kerho et al. [9]. Also Lin [7] observed the Drag reducing and Lift increasing effects of micro VGs. Wendt [10] investigated an array of VGs in an experiment where the VGs were mounted in a fashion to generate counter-rotating vortices. Furthermore, the experimental and numerical studies carried out by Ashill et al. [11,12] revealed a successful delaying of the shock-induced separation by the implementation of Sub Boundary-Layer Vortex Generators (SBVG). Two series of investigations were presented at both zero and adverse streamwise pressure gradients, where vortex decay and device drag were reasonably well predicted by the RANS based computations.

The effect of VGs on a 1 MW Wind Turbine was investigated by Øye [13] and Miller [14], who compared the measured power curves on a (however optimistically designed) wind turbine with and without VGs. Though quite rough methods for the optimization of the VG design were employed, these studies showed that in the case under investigation the VGs on average increased the output power for nearly all wind speeds. Adding VGs to wind turbine blades is often a simple measure to improve the performance of a rotor, though great care needs to be taken not to deteriorate the performance of the wind turbine, or, even worse, to change the aeroelastic performance adversely so that the wind turbine becomes destabilized. This can significantly reduce the lifetime of the wind turbine, which further highlights the need of proper models for VGs.

Many models for the generated vortices have been presented over the years. Theoretical models include, for example, the one by Smith [15] and a model presented by Velte et al. [5] that was developed and applied to show the helical symmetry of the vortices generated by a passive rectangular vane-type vortex generator. As for more practically and numerically oriented models incorporated into codes, most are variants of the practical BAY-model by Bender et al. [16], which introduces body forces using source terms in the Navier-Stokes equations to simulate the presence of a vane.

The fact that the vortices produced by vortex generators possess helical symmetry means, in effect, that the streamwise profiles $\left(u_{z}\right)$, along the longitudinal vortex axis and the rotational $\left(u_{\theta}\right)$ flows are inter-related by a simple linear relation based on the helical shape of the vorticity lines [5, 17]. Further, previous experimental work by Velte [18] examines the downstream vortex evolution behind a cascade of vortex generators producing counter-rotating vortices in a boundary layer of negligible streamwise pressure gradient. The model parameters are all seen to vary linearly in the downstream direction. Based on the experimental observations of [18], the vortices generated by vortex generators have been observed 


\section{COMPUTATIONAL CONFIGURATION}

In this study, steady state simulations were carried out and compared to experimental observations. These computations were performed using the EllipSys3D code, see Michelsen [20] and Sørensen [21]. This in-house CFD code is a structured finite-volume flow solver using, in this work, Reynolds-Averaged Navier-Stokes equations. The pressure/velocity coupling is ensured using the SIMPLE algorithm. The convective terms are discretized utilising the third order Quadratic Upstream Interpolation for Convective Kinematics (QUICK), [22]. For these computations the $k-\omega S S T$ (Shear Stress Transport) turbulence model by Menter [23] was used. A successful validation of steady RANS simulations against measurements 
is the one carried out by Allan [24], where the flow field about a single VG on a flat plate with two different vane angleof-attack was computed using a flow solver code developed at NASA. The results of these simulations were compared to experimental data and showed that the CFD and experiments agreed well except for short distances downstream of the vane.

Figure 2 illustrates the computational setup with the current setting consisting of a single VG on a flat plate. The actuator is a rectangular vane with a length two times the VG height. The computational domain with dimensions normalized with the VG height is also given in Figure 2, as also described more in detail in [25]. The thickness of the vane is constant and the VG was positioned directly on the wall at a position where the boundary layer thickness is equal to the VG height. The data were extracted in 5 spanwise planes, normal to the test section floor and located 5, 7.5, 10, 12.5 and 15 VG heights downstream of the vortex generator trailing edge.

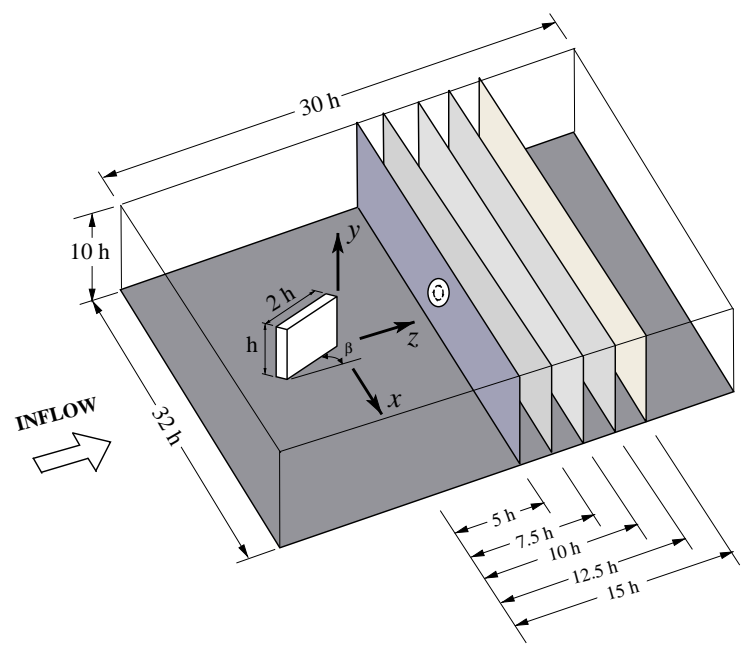

Figure 2. Computational domain (not to scale).

The computational setup was designed to mimic the one of the experiments [18]. The VG angle of attack to the oncoming flow is $\beta=20^{\circ}$ and the Reynolds number based on VG height $h=0.25 \mathrm{~m}$ is $R e=1700$, using an inflow velocity $U_{\infty}=$ $1 \mathrm{~ms}^{-1}$ and a density of $1 \mathrm{kgm}^{-3}$. At the bottom wall and on the VG surface no-slip conditions were specified, while the upstream and lid part of the domain were specified as inflow conditions with prescribed velocity according to the undisturbed velocity. The two side faces of the domain were specified as slip/symmetry conditions, while the downstream plan was set as an outlet assuming fully developed flow. At the inlet the turbulence is set to give very low eddy viscosity, and the turbulence seen by the VG is generated by the developing boundary layer along the wall surface.

In the experiments, the turbulence intensity at the inlet from laser Doppler anemometry (LDA) measurements was found to be $13 \%$ where the turbulence was generated by a grid at the inlet. The boundary layer thickness at the position of the vortex generators was estimated from LDA measurements to be approximately $\delta_{V G}=25 \mathrm{~mm}$. The actuators were rectangular vanes of the same height as the local boundary layer thickness, $h=\delta_{V G}$, with a length of $2 h$. The computations have been carried out based on the experimental inflow profile illustrated in Figure 3. This figure represents the measured (Stereoscopic Particle Image Velocity) turbulent boundary layer profile without VG at the position where the VG was later mounted. A more complete characterization of the inlet conditions can be found in the $\mathrm{PhD}$ thesis of Velte [26].

The computational setup of the CFD simulations consists of a block structured mesh of 18 million cells with the first cell height $(\Delta \mathrm{z} / \mathrm{h})$ of $1.5 \times 10^{-6}$ normalized by the VG height. In the immediate vicinity of the vane, the mesh has $5 \times 10^{6}$ cells, while the mesh downstream the VG for capturing the wake has approximately $2.5 \times 10^{6}$ cells. In order to resolve the boundary layer, cell clustering has been used close to the wall and the dimensionless distance from the wall is 


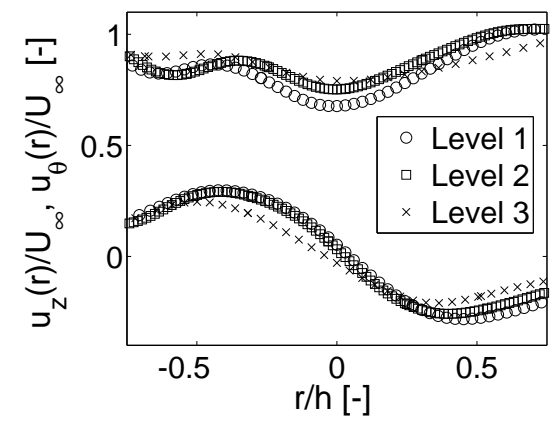

Figure 4. Axial and azimuthal velocity profiles for three different mesh sizes of the computations..

\section{RESULTS}

The analysis of helical symmetry was performed on computational results extracted in the cross planes positioned at $z / h=5,7.5,10,12.5$ and $15 \mathrm{VG}$ heights downstream of the trailing edge of the VG. The reason for the analysis not to be performed at positions closer to the VG is that the wake in this range is still forming and therefore has not yet developed the helical symmetry (see also $[18,26]$ ). An angle of attack of $\beta=20^{\circ}$ of the VG to the oncoming flow was chosen for the computations and subsequently compared with the wind tunnel experiments and the analytical model in [18]. The vane angle is close to the optimum found by Godard et al. [28] in a parametric study optimizing separation control. The extraction of velocities from the computations was conducted in a similar way to the experimental procedure described in 
[5], in planes normal to the section wall downstream of the VG and applying polar coordinates $(z, r, \theta)$ to the vortex with the origin at the vortex center.

\subsection{Testing of helical symmetry}

The first necessary step is to check whether the simulation results fulfill helical symmetry. In order to perform this test, the three velocity components were extracted along lines passing through the center of the primary vortex at four angles of $\Theta\left(0^{\circ}, 45^{\circ}, 90^{\circ}\right.$ and $\left.135^{\circ}\right)$ to cover the full rotational variations of the vortex, see Figure 5. The cylindrical coordinate system used to describe these variations across the vortex core is presented in Figure 5, with the center always located at the vortex core center. The origin of the $z$-axis is located at the upper edge of the VG trailing edge.

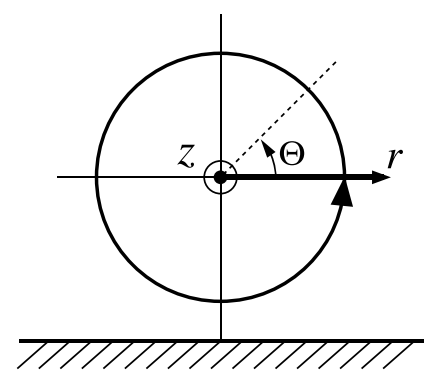

Figure 5. Polar coordinate system centered at the vortex core center.

The solid curves in Figure 6 illustrate the axial and azimuthal velocity profiles (upper curves are the axial velocity profile $u_{z}$ and lower the azimuthal one $u_{\theta}$ ) for each plane position where the data is extracted along a horizontal line through the vortex center. These curves are compared to what is obtained from the right-hand side of equation (1), finding $l$ by least squares fitting of the residual, see [18]. The two data sets overlap well for all plane positions, confirming that the computational results fulfill the criterion for helical symmetry just like the experimental ones do. Note that the analysis has been carried out only on the right side, due to a perturbing secondary vortex appearing on the left side yielding an asymmetric velocity profile [5].

$$
u_{z}=u_{0}-\frac{r}{l} u_{\theta}
$$

Figure 7 displays the stream-wise evolution of the helical parameters that directly result from this analysis: vortex convection velocity $\left(u_{0}\right)$, circulation $(\Gamma)$, helical pitch $(l)$ and vortex core radius $(\varepsilon)$ for the wind tunnel experiments and computational simulations. The error bars illustrate the error of the linear fit to the data. The local flow characteristic $u_{0}$ was found directly from the lowest value of the axial velocity wake profile in the vortex core and the helical pitch $l$ was obtained from least-squares fitting of equation (1). The circulation has been calculated from integration as the flux of vorticity across a surface enclosed by a curve described by the vortex radius. The vortex core radius $\varepsilon$ has been obtained as the radius of the maximum value of the azimuthal velocity for each plane position. For a better comparison between the experimental and computational results, both vortex radius and helical pitch have been normalized by the VG height $h$ and averaged across the azimuthal coordinate $\Theta$. To illustrate the helical parameter dependency on theta, Figure 8 was represented. This figure provides the CFD results of the evolution downstream of the VG for the helical pitch $l$ and the vortex core radius $\varepsilon$ at different angles of $\Theta$. The convection velocity has been normalized by the free stream-velocity $U_{\infty}$ and the circulation by the product $U_{\infty} h$. Since the axial and azimuthal velocities are observed to be self-similar, it is expected that $u_{0}$ and $l$ also vary linearly along the downstream path, which is indeed observed for both the experiments and 

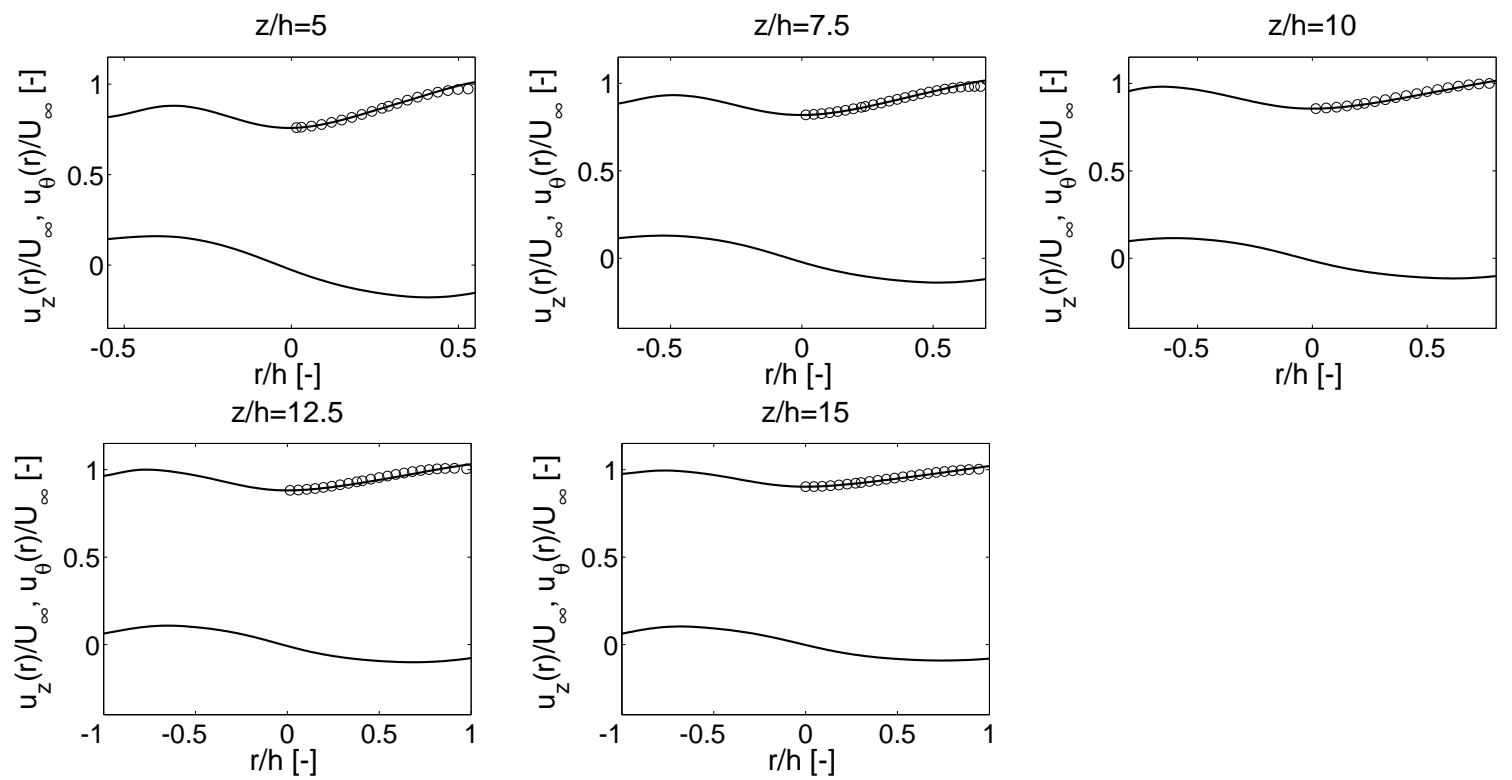

Figure 6. CFD velocity profiles of embedded vortices generated by a VG for a device angle $\beta=20^{\circ}$ and $\Theta=0^{\circ}$. The negative $r / h$ values represent the $r / h$ axis for $\Theta=180^{\circ}$. Upper values are the axial velocity profile $u_{z}$ and lower the azimuthal one $u_{\theta}$ normalized by $U_{\infty}$. For positive $r / h, C F D$ values of $u_{z}$ are compared to the right-hand side of equation (1) calculated using the computational values $u_{0}$ and $u_{\theta}$ (o).

the simulations in Figure 7. As expected, the only factor which does not vary along the plane positions is the circulation, which should naturally be close to constant in a system of low viscous dissipation.

In fact, all helical parameters are seen to vary linearly with the downstream distance, both for experiments and simulations. Further, the general trends agree in the downstream evolution of all parameters, though the absolute values differ between the quantities. As pointed out earlier, the vortex strength is larger in the simulations and the vortex core size is also somewhat larger and varies with a steeper gradient in the downstream direction. The normalized convection velocity $u_{0} / U_{\infty}$ (minimum axial velocity at the center of the vortex core divided by the free stream-velocity) in the downstream direction was also observed to be smaller in the measurements for the positions closer to the VG, while in the positions far away from the trailing edge of the VG it is larger in the measurements in comparison with the CFD results, see Figure 7. This means that the axial velocity induction caused by the helical vorticity lines is stronger in the measurements far away from the VG. The relation between the convection velocity and the helical pitch is not as straightforward though, since the strength of the vorticity lines may vary.

\subsection{Testing of wake self-similarity}

Figures 9 display the axial $\left(u_{z}\right)$ and azimuthal $\left(u_{\theta}\right)$ velocity profiles of the measurements (dash-dot lines) and computational data (solid lines). The left column shows a combined plot of the axial and azimuthal velocity profiles for all downstream positions. The mid and right columns show the self-similarity scaled axial and azimuthal velocity profiles, respectively. In the case of the computations, the velocity profiles have been represented at the plane positions $z / h=5-15$, while the experimental profiles have been represented at $z / h=5-13$, according with [18]. Note that these profiles all collapse nicely except on the left side of these profiles, in particular for the axial velocity, where the secondary vortex perturbation appears.

From visual inspection of the left column in Figure 9, it becomes apparent that the axial and azimuthal velocity profiles in the vortex core do not change significantly for the various angles $\Theta$. Of course, the presence of the wall and the emergence of the perturbing secondary structure create distortions in the outer regions of the core, which is particularly observable on 

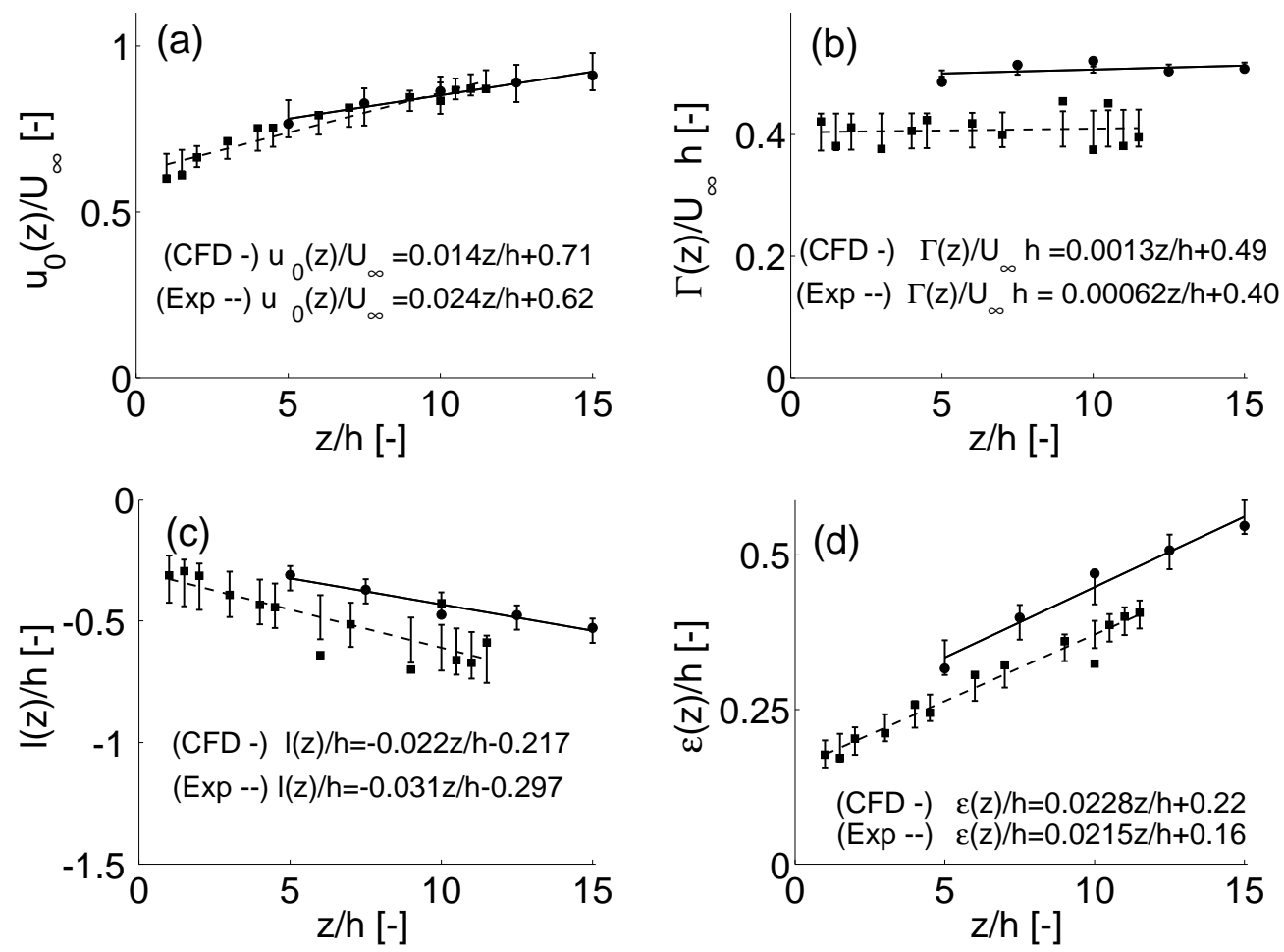

Figure 7. Comparison between CFD and experimental results of the downstream evolution of the characteristic vortex parameters.
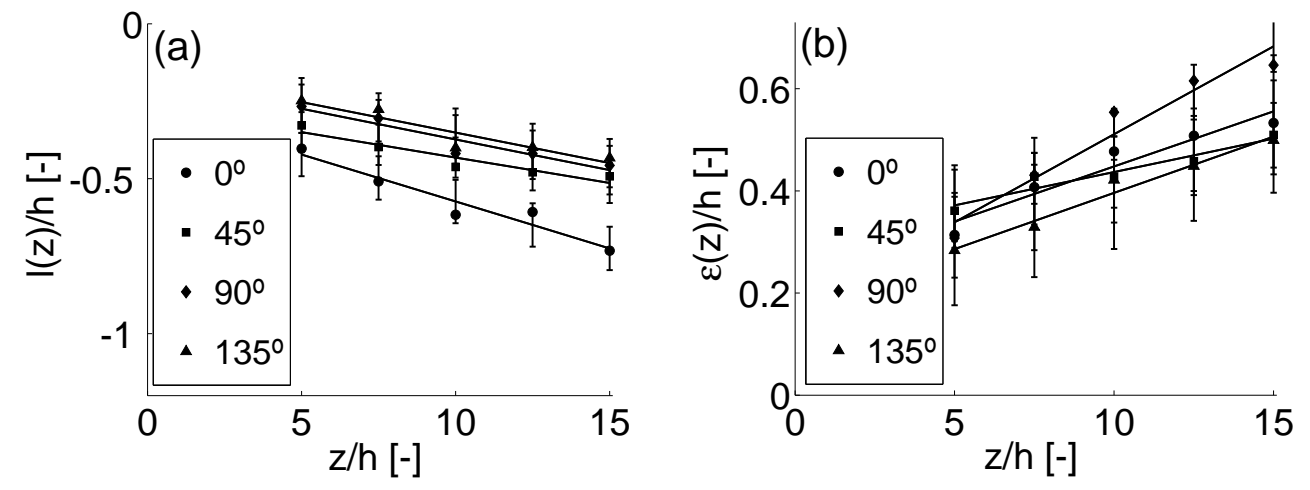

Figure 8. CFD results of the downstream evolution of the helical pitch (a) and the vortex radius (b) at different angles of $\Theta: 0^{\circ}, 45^{\circ}$, $90^{\circ}$ and $135^{\circ}$.

the left side of the plots for $\Theta=45^{\circ}, 90^{\circ}$ and $135^{\circ}$. The computational results follow the same trend of the experiments, though some features are pronounced to greater or lesser extent. The effects of the secondary perturbing vortex are more clearly visible on the left side of the axial velocity profiles of the experimental results for $\Theta=0^{\circ}$. Moreover, the speed-up in the azimuthal velocity close to the wall for $\Theta=90^{\circ}$ is larger in the experiments compared to the computational profiles, which is consistent with the stronger induction further downstream. These anomalies can be attributed to the vortex core strength being larger in the computations, see Figure 7, and the vortex core distance to the wall being smaller in the wind tunnel experiments. More specifically, the distance from the center of the vortex to the wall in the case of the computations corresponds to 0.84 times the VG height, measured in a plane perpendicular to the wall and five times the device height downstream of the VG. While in the experiments, it was observed that the distance from the center of the vortex to the wall 
$\Theta=0^{0}$
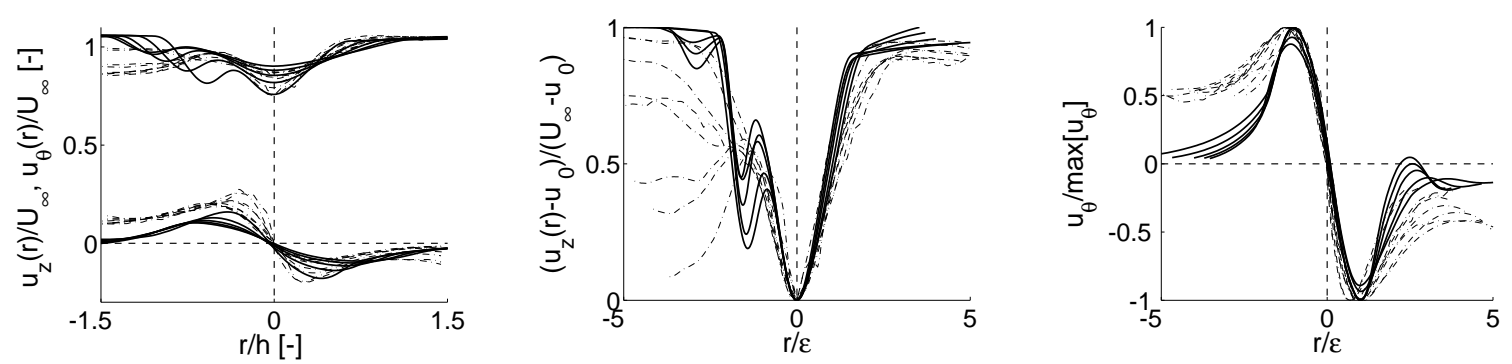

$\Theta=45^{0}$
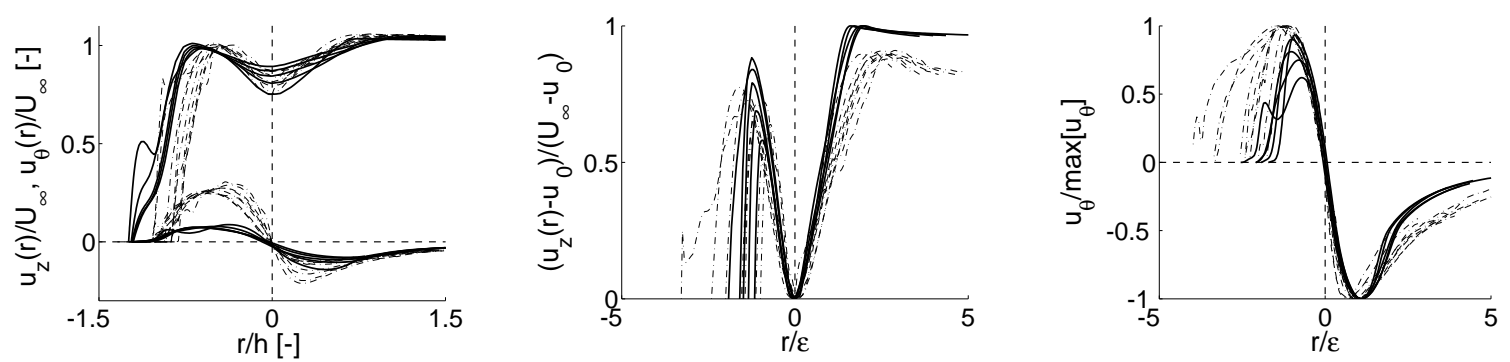

$\underline{\Theta}=90^{0}$
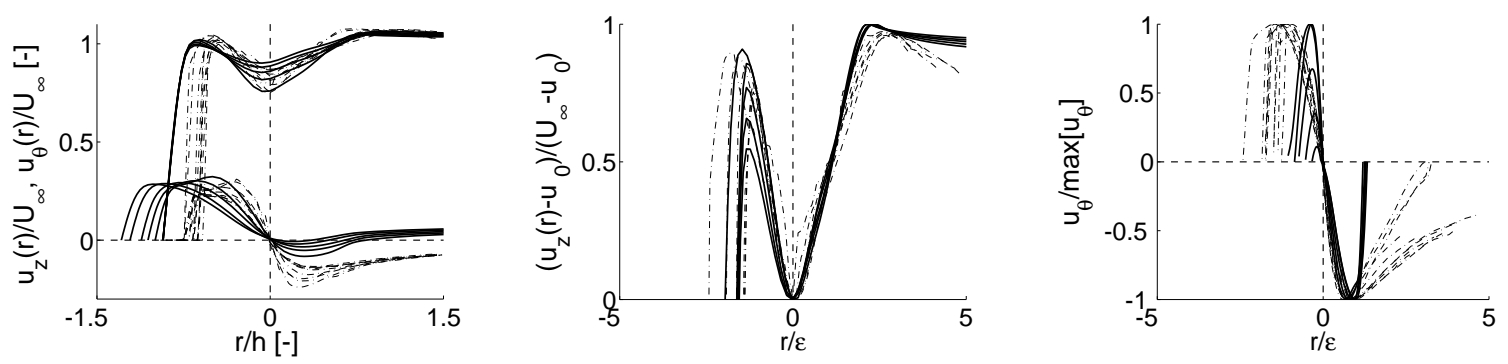

$\underline{\Theta}=135^{0}$
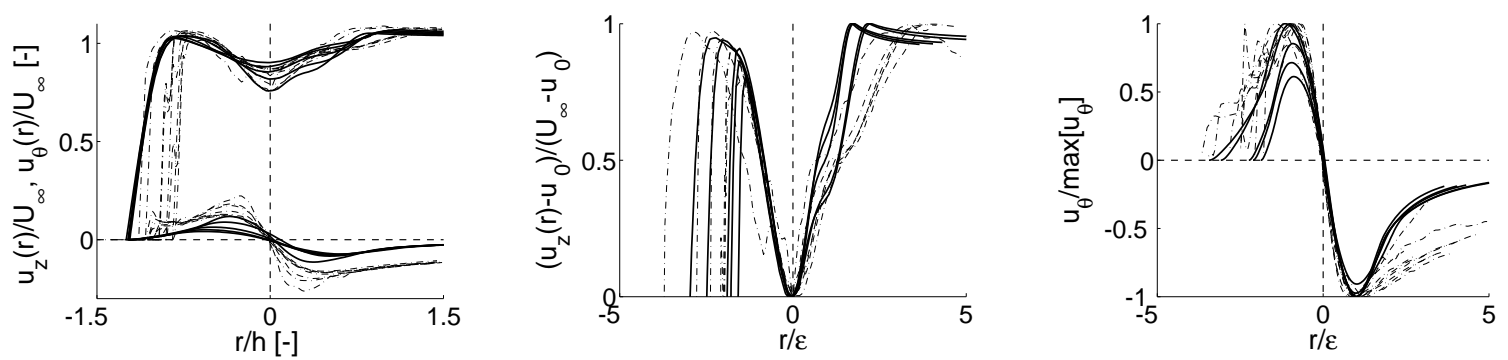

Figure 9. CFD (solid lines) and experimental (dash-dot lines) velocity profiles for various angles $\Theta$ at different plane positions downstream of the VG. The left side shows the axial $\left(u_{z}\right)$ and azimuthal $\left(u_{\theta}\right)$ profiles and the middle and right sides show the axial and azimuthal profiles respectively, scaled by self-similarity variables.

study shown quantitatively that CFD can reproduce the same kind of trends that are observed in measurements. Note that, apart from the highly complex viscous interactions with secondary structures and the wall, the current model used can describe the complete flow characteristics of the VG wake. This kind of comparison has previously only been done in a qualitative fashion or only quantitatively to some extent. 


\section{CONCLUSIONS}

Vortices generated by a passive rectangular vane-type vortex generator of the same height as the local boundary layer thickness above a test section wall have been studied. CFD simulations at Reynolds number $R e=1700$ based on the VG height $h=0.25 \mathrm{~m}$ and free stream velocity $U_{\infty}=1 \mathrm{~ms}^{-1}$ have been carried out using the RANS method and compared with wind tunnel experimental data and an analytical model.

The vortex generated by the VG shows self-similar behaviour for both the axial and azimuthal velocity profiles. It has been proven based on the computer simulated data from five cross-plane positions $\mathrm{z} / \mathrm{h}=5,7.5,10,12.5$ and 15 downstream of the trailing edge of a VG with an angle of attack $\beta=20^{\circ}$ to the oncoming flow, just as in the experiments. The computational results show relatively good agreement with the self-similarity shown in the experiments, both displayed in Figure 9, and also present in [18]. The trends of the characteristic helical vortex parameters in the computational results match the experimental observations reasonably well (Figure 7) considering the limitations of the turbulence models for reproducing the embedded vortices produced by vane-type VGs and the applied inlet conditions.

From the point of view of self-similarity, computational simulations are able to reproduce the physics of the vortex generated by a rectangular VG with reasonably good reliability. Furthermore, the helical symmetry has been also tested and verified based on the computational data.

As CFD methods are the most common ones applied in optimizing VGs on wind turbine blades, the detailed information provided by the investigations of the present study can be used as a guidance for successful implementation of VGs on a wind turbine blade and to investigate parametric dependencies of the VGs on different boundary layers. Due to the vast range of parameters inherent in the problem (angle, interspacing, orientation, VG height and length etc.) an exhaustive understanding of the flow is required to be applied properly, in which the application of this simplifying model can be helpful. Note that the current model applied can describe the complete flow characteristics of the VG wake (except for the viscous wall/secondary structures interactions) in a physical fashion and facilitates the validation of computational results. To the knowledge of the authors, this kind of comparison has previously only been done in a qualitative fashion or only quantitatively to some extent, i.e., only covering some aspects of the VG induced wake.

\section{ACKNOWLEDGEMENTS}

UF-G is very grateful to E. Zulueta of the University College of Engineering Vitoria-Gasteiz (UPV-EHU, Spain) for his helpful remarks. UF-G is also thankful to the Government of the Basque Country by the financial support of this work through the SAIOTEK research plan (S-PE11UN112). CMV and NNS were supported by EUDP-2009-II-Grant Journal No. 64009-0279, which is also gratefully acknowledged. Computations were made by use of Thyra PC-cluster at DTU Ris $\varnothing$ Campus.

\section{REFERENCES}

1. Taylor HD. Application of vortex generator mixing principles to diffusers. Research Department Concluding Report No. R-15064-5. United Aircraft Corporation, East Hartford, Connecticut, 1948.

2. Taylor HD. Design criteria for and applications of the vortex generator mixing principle. Research Department Report No. M-15038-1. United Aircraft Corporation, East Hartford, Connecticut, 1948.

3. Taylor HD. Summary report on vortex generators. Research Department Report No. R-05280-9. United Aircraft Corporation, East Hartford, Connecticut, 1950.

4. Schubauer GB, Spangenber WG. Forced mixing in boundary layers. Journal of Fluid Mechanics 1960; 8:10-32. 
5. Velte CM, Hansen MOL, Okulov VL. Helical structure of longitudinal vortices embedded in turbulent wall-bounded flow. J. Fluid Mech 2009; 619, 167-177.

6. van Rooij RPJOM, Timmer WA. Roughness sensitivity considerations for thick rotor blade airfoils. Journal of Solar Energy Engineering-Transactions of the ASME 2003; 125(4):468-478 DOI: 10.1115/1.1624614.

7. Lin JC. Review of research on low-profile vortex generators to control boundary layer separation. Progress in Aerospace Sciences 2002; 38: 389-420.

8. Pearcey HH. Shock induced separation and its prevention by design and boundary layer control. In Boundary Layer and Flow Control (ed. G. V. Lachmann) Pergamon Press 1961, 2, pp. 1166-1344.

9. Kerho M, Huctcherson S, Blackwelder RF, Liebeck RH. Vortex Generators used to control laminar separation bubbles. Journal of Aircraft 1993; 30(3): 315-319.

10. Wendt BJ. Parametric study of vortices shed from airfoil vortex generators. AIAA Journal 2004; 42: 2185-2195.

11. Ashill PR, Fulker JL, Hackett KC Research at DERA on sub boundary layer vortex generators (SBVGs). 39th Aerospace Sciences Meeting and Exhibit, AIAA 8-11 January 2001, Reno, Nevada.

12. Ashill PR, Fulker JL, Hackett KC Studies of flows induced by sub boundary layer vortexgenerator s (SBVGs)). 40th Aerospace Sciences Meeting and Exhibit, AIAA 14-17 January 2002, Reno, Nevada.

13. Øye S. The effect of vortex generators on the performance of the ELKRAFT 1000kW turbine. 9th IEA Symposium on Aerodynamics of Wind Turbines 1995, Stockholm, Sweden, ISSN 0590-8809.

14. Miller GE. Comparative performance tests on the Mod-2, 2.5-MW wind turbine with and without vortex generators. DOE/NASA Workshop on Horizontal Axis Wind Turbine Technology 1984, Cleveland, OH, United States, 8-10.

15. Smith FT. Theoretical prediction and design for vortex generators in turbulent boundary layers. Journal of Fluid Mechanics. 1994; 270: 91-131.

16. Bender EE, Anderson BH, Yagle PJ. Vortex generator modeling for Navier-Stokes codes. Proc. 3rd ASME/JSME Joint Fluids Engineering Conference 1999, San Francisco, California, USA.

17. Alekseenko SV, Kuibin PA, Okulov VL. Theory of Concentrated Vortices. An Introduction. (1st edn), SpringerVerlag, Berlin Heidelberg, 2007, Chap. 1.

18. Velte CM. A vortex generator flow model based on self-similarity. AIAA Journal (doi: 10.2514/1.J051865), 51(2):526$529,2013$.

19. White FM. Viscous Fluid Flow, (2nd edn), McGraw-Hill, Singapore, 1991, pp. 470-481.

20. Michelsen JA. Basis3D- a platform for development of multiblock PDE solvers. Technical Report AFM 94-06, Technical University of Denmark, Dept. of Mechanical Engineering, 1994.

21. Sørensen NN. General purpose flow solver applied to flow over hills. Technical Report Risoe-R-827(EN), Risoe National Laboratory, 1995.

22. Khosla K, Rubin SG. A diagonally dominant second-order accurate implicit scheme. Computer Fluids 1974; 207-209.

23. Menter FR. Zonal two equation $k-\omega$ turbulence model for aerodynamic flows. AIAA 24th Fluid Dynamics Conference. Orlando, Florida, July 1993.

24. Allan BG, Chung-Sheng Yao C-S, Lin JC. Numerical simulations of vortex generators and jets on a flat plate. 1st Flow Control Conference, AIAA 24-27 June 2002, St. Louis, Missouri.

25. Fernández U, Réthoté P-E, Sørensen NN, Velte CM, Zahle F, Egusquiza E. Comparison of four different models of vortex generators. Proceedings of EWEA - European Wind Energy Conference, Copenhagen, 16-19 2012.

26. Velte CM. Characterization of Vortex Generator Induced Flow. PhD Thesis, Technical University of Denmark (DTU), Lyngby, Denmark 2009.

27. Vinokur M. On one-Dimensional Stretching Functions for Finite-Difference Calculations. Journal of Computational Physics 1983 50: 215-234.

28. Godard G, Stanislas M. Control of a decelerating boundary layer. Part 1: Optimization of passive vortex generators. Aerospace Science and Technology 2006 10: 181-191. 


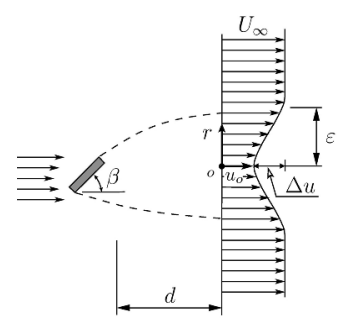

Wake velocity profile of a single VG at a distance $\backslash$ emph $\{\mathrm{d}\}$ from the trailing edge of the vane with free

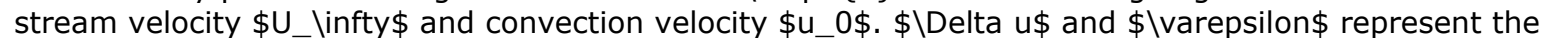
characteristic velocity scale and the characteristic shear-layer width, respectively. $297 \times 420 \mathrm{~mm}(300 \times 300 \mathrm{DPI})$ 


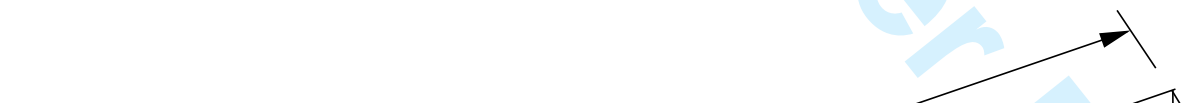




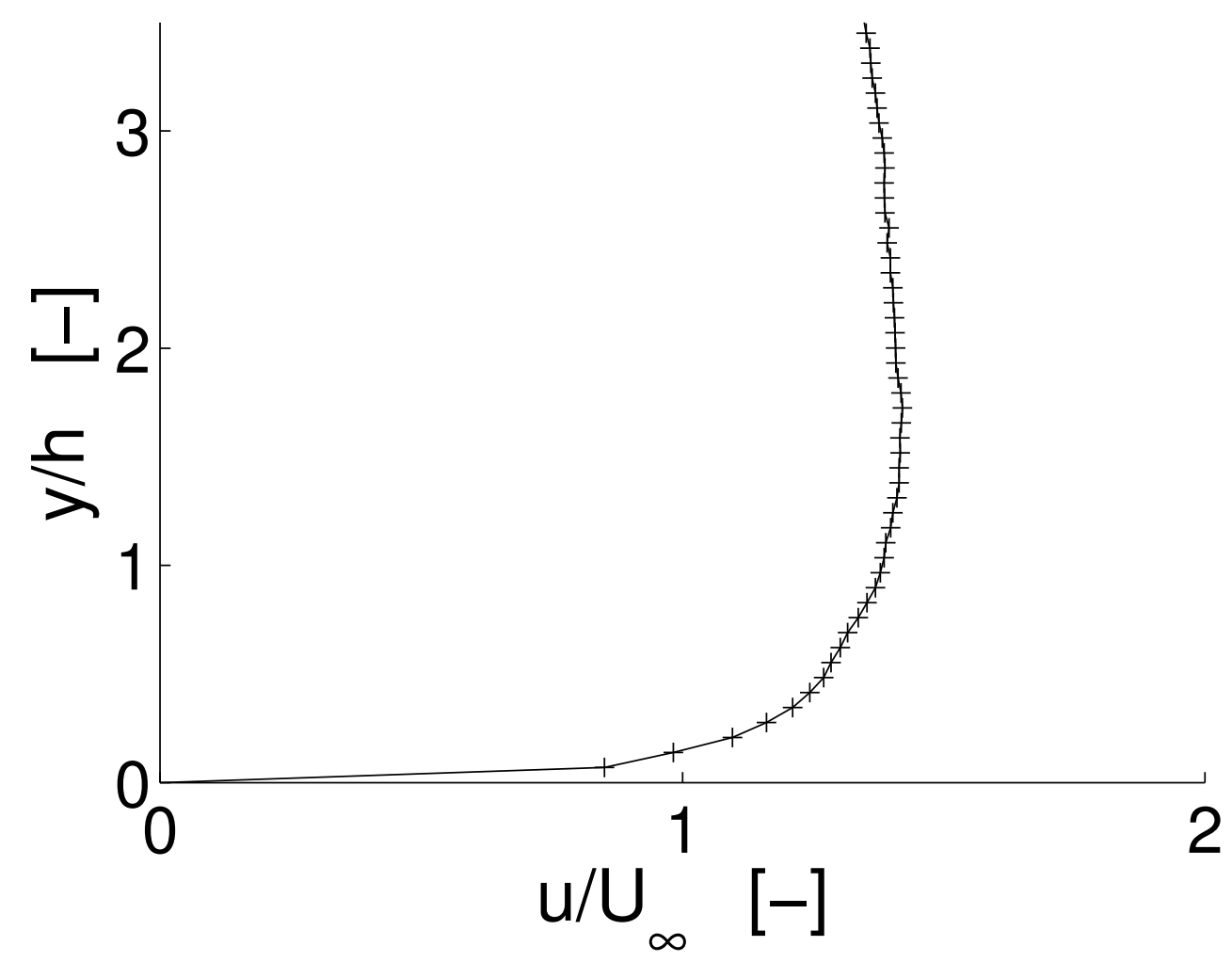

John Wiley \& Sons 


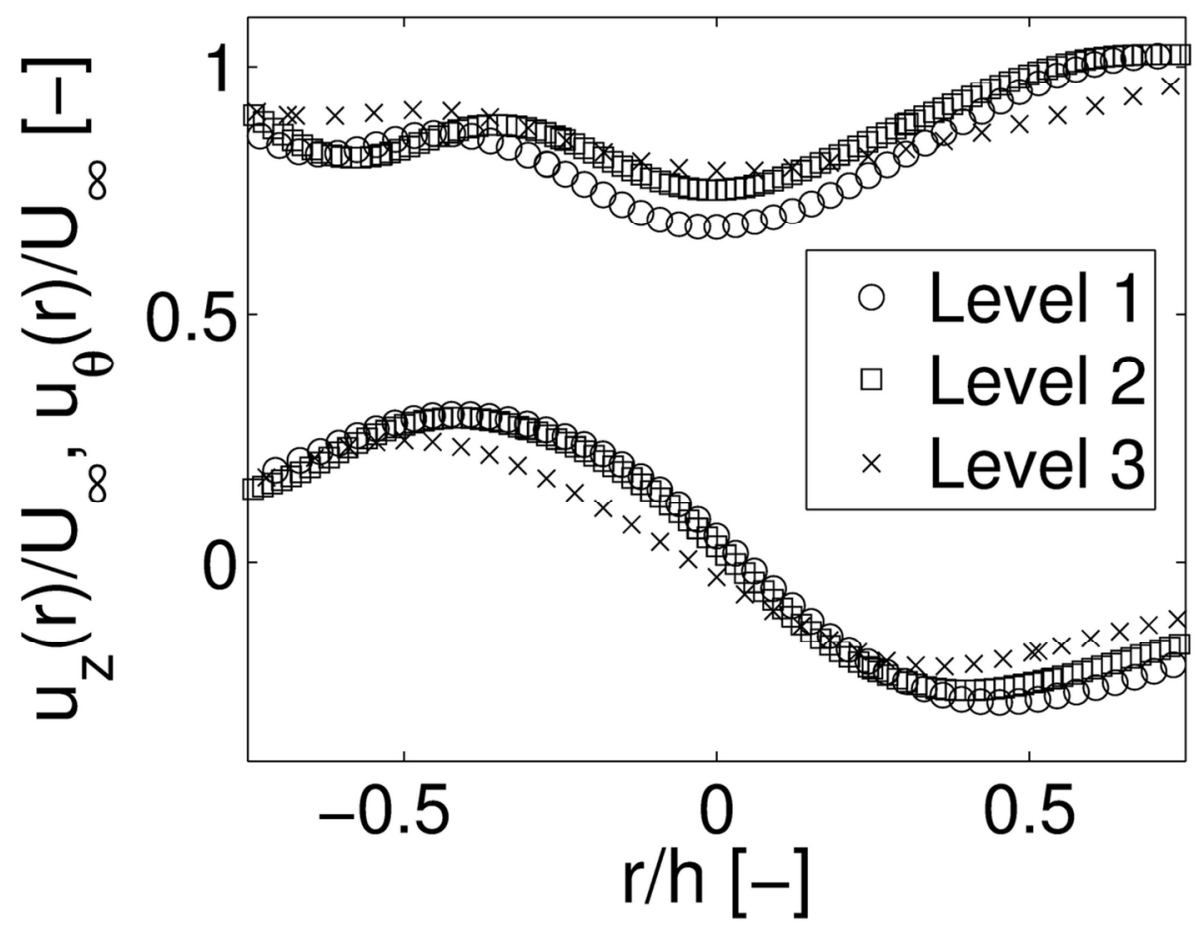

Axial and azimuthal velocity profiles for three different mesh sizes of the computations. $111 \times 83 \mathrm{~mm}(300 \times 300 \mathrm{DPI})$ 
1
2
3
4
5
6
7
8
9

10

11

12

13

14

15

16

17

18

19

20

21

22

23

24

25

26

27

28

29

34

35

36

37

38

39

40

41

42

43

44

45

46

47

48

49

50

51

52

53

54

5

5
5
5

58

60

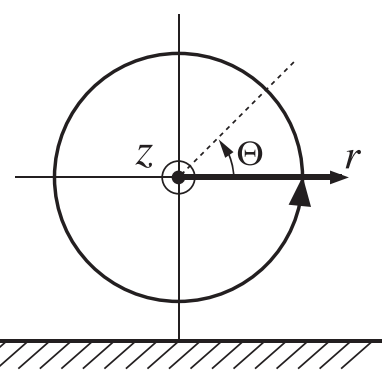

John Wiley \& Sons 


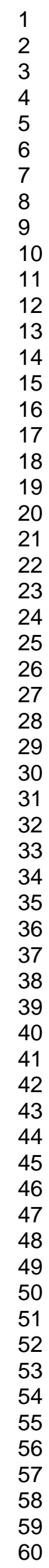

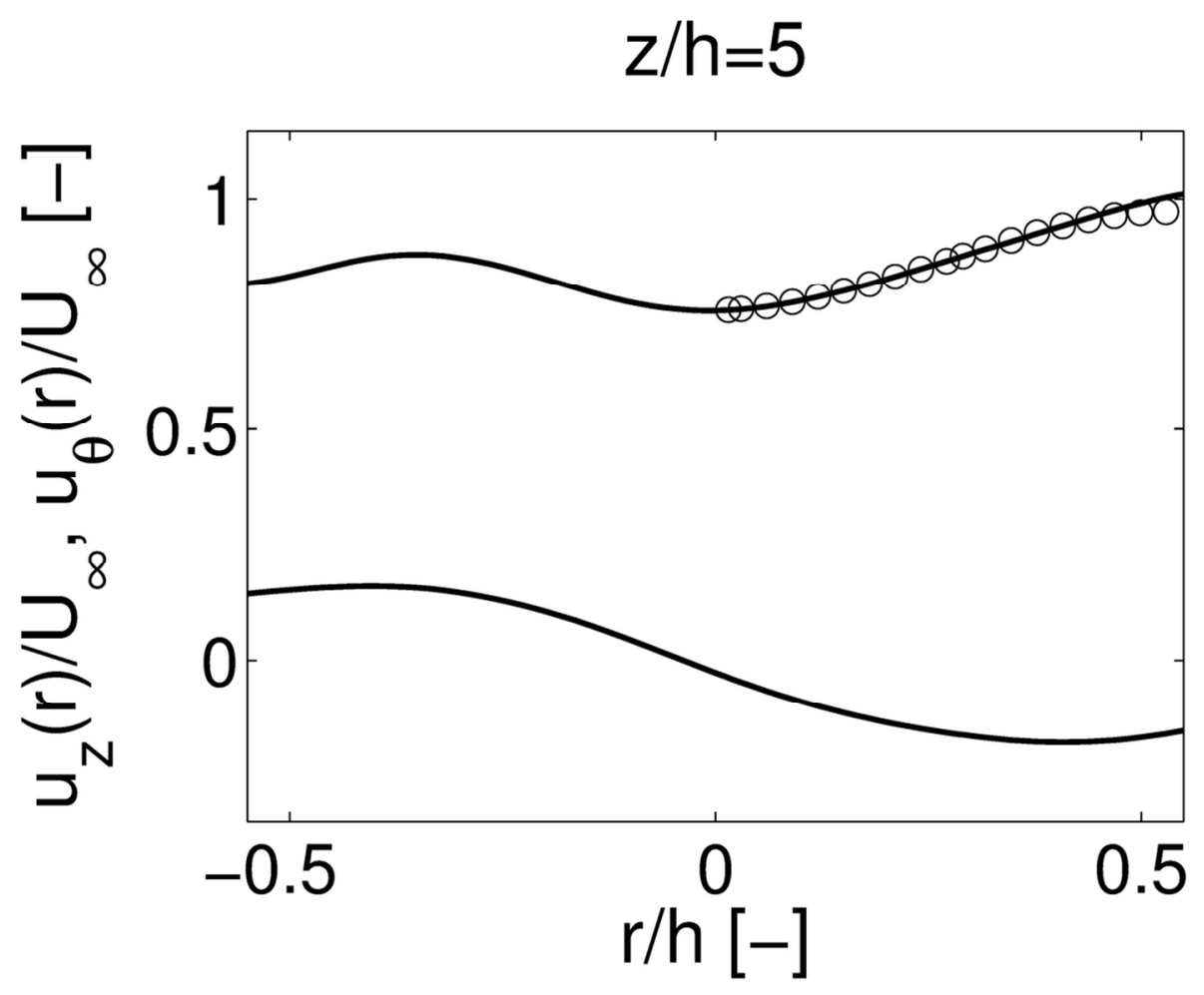

$111 \times 83 \mathrm{~mm}(300 \times 300 \mathrm{DPI})$

John Wiley \& Sons 
$\mathrm{z} / \mathrm{h}=7.5$

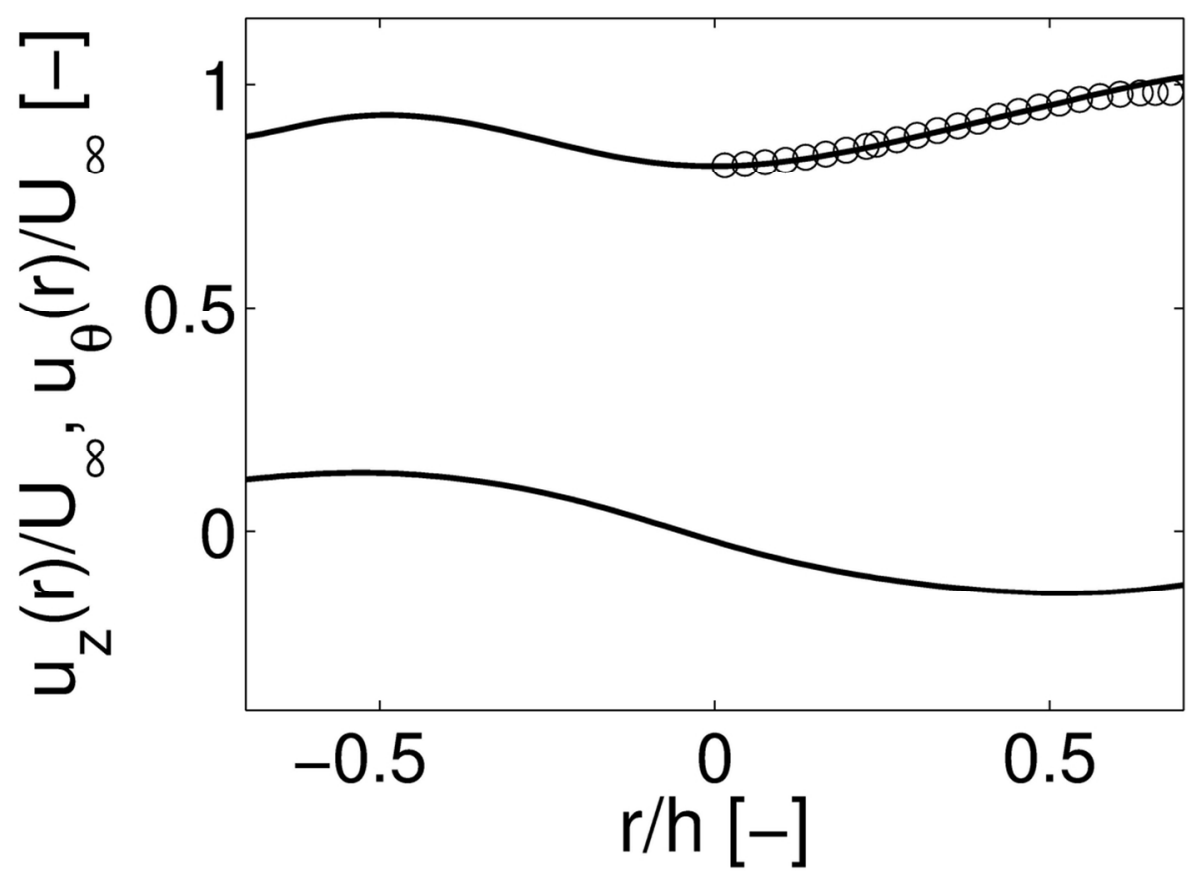

$111 \times 83 \mathrm{~mm}(300 \times 300$ DPI $)$ 


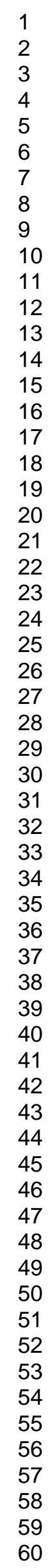

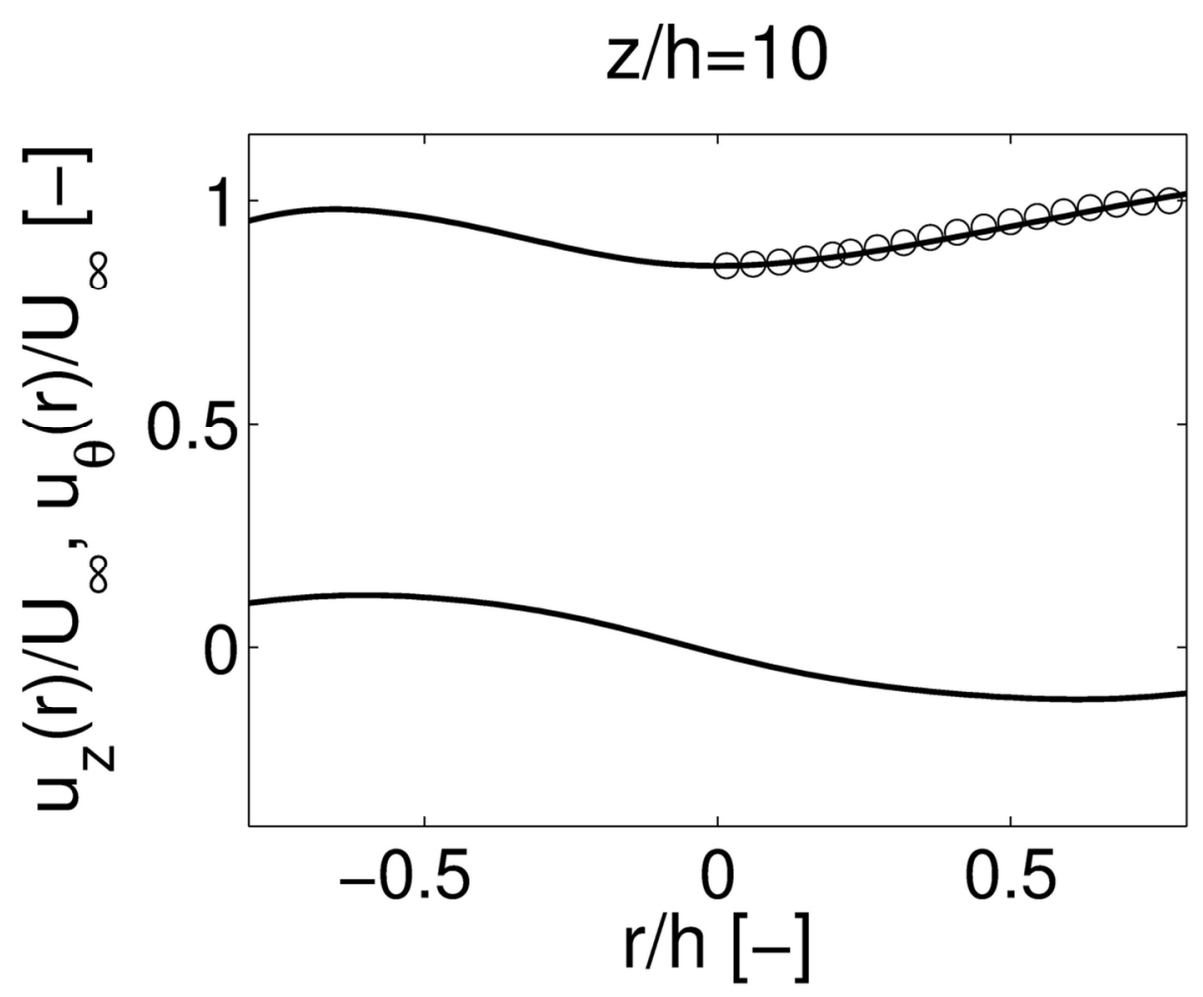

$111 \times 83 \mathrm{~mm}(300 \times 300 \mathrm{DPI})$

John Wiley \& Sons 
$z / h=12.5$

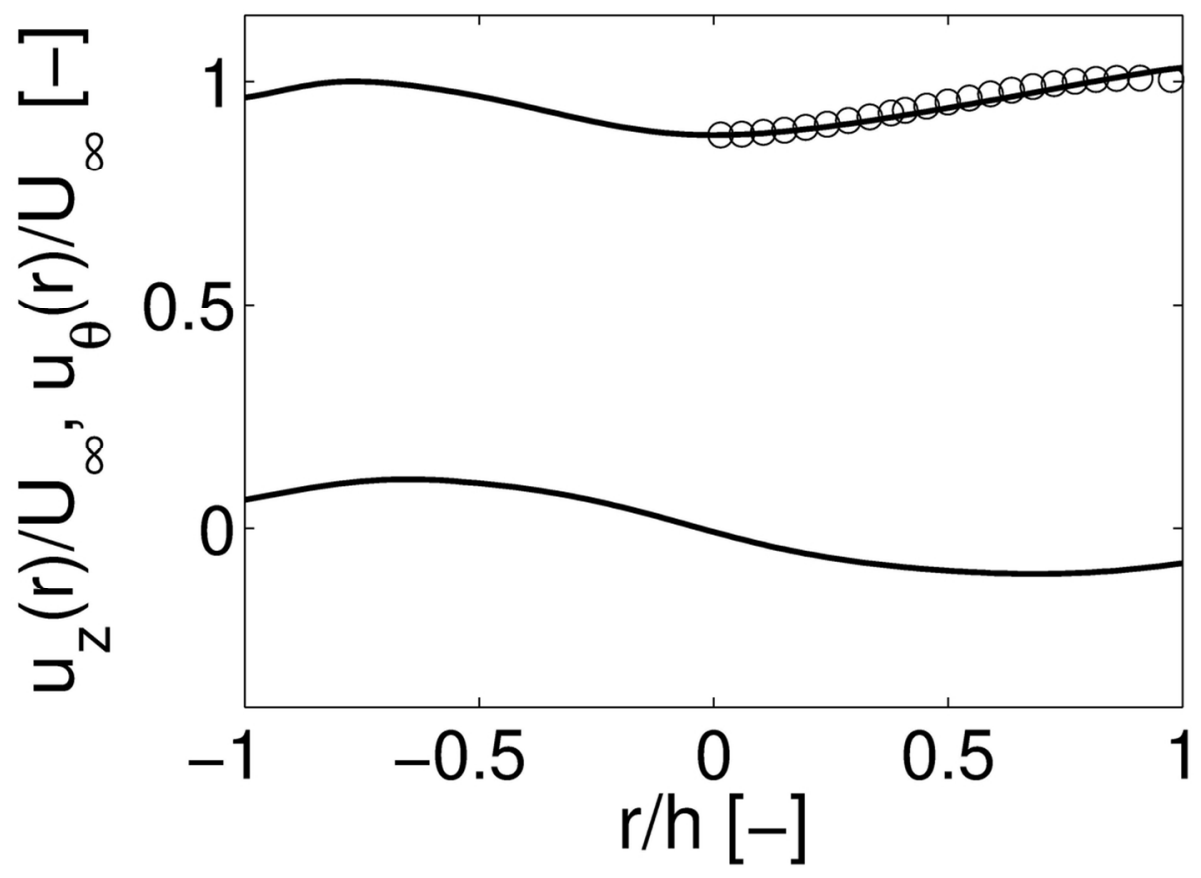

$111 \times 83 \mathrm{~mm}(300 \times 300 \mathrm{DPI})$ 
CFD velocity profiles of embedded vortices generated by a VG for a device angle $\$ \backslash$ beta $=20^{\wedge} 0 \$$ and $\$ \backslash T$ Theta $=0^{\wedge} 0 \$$. The negative $\$ r / h \$$ values represent the $\$ r / h \$$ axis for $\$ \backslash$ Theta $=180^{\wedge} 0 \$$. Upper values are the axial velocity profile $\$ u_{-} z \$$ and lower the azimuthal one $\$ u_{-} \mid$theta $\$$ normalized by $\$ U_{-}\{\backslash$ infty $\} \$$. For positive $\$ r / h \$, C F D$ values of $\$ u \_z \$$ are compared to the right-hand side of equation ( $\backslash r$ ref $\{$ eqn 1$\}$ ) calculated using the computational values $\$ \mathrm{u}_{-} 0 \$$ and $\$ \mathrm{u}_{-} \mid$theta $\$(0)$.

$111 \times 83 \mathrm{~mm}(300 \times 300 \mathrm{DPI})$ 


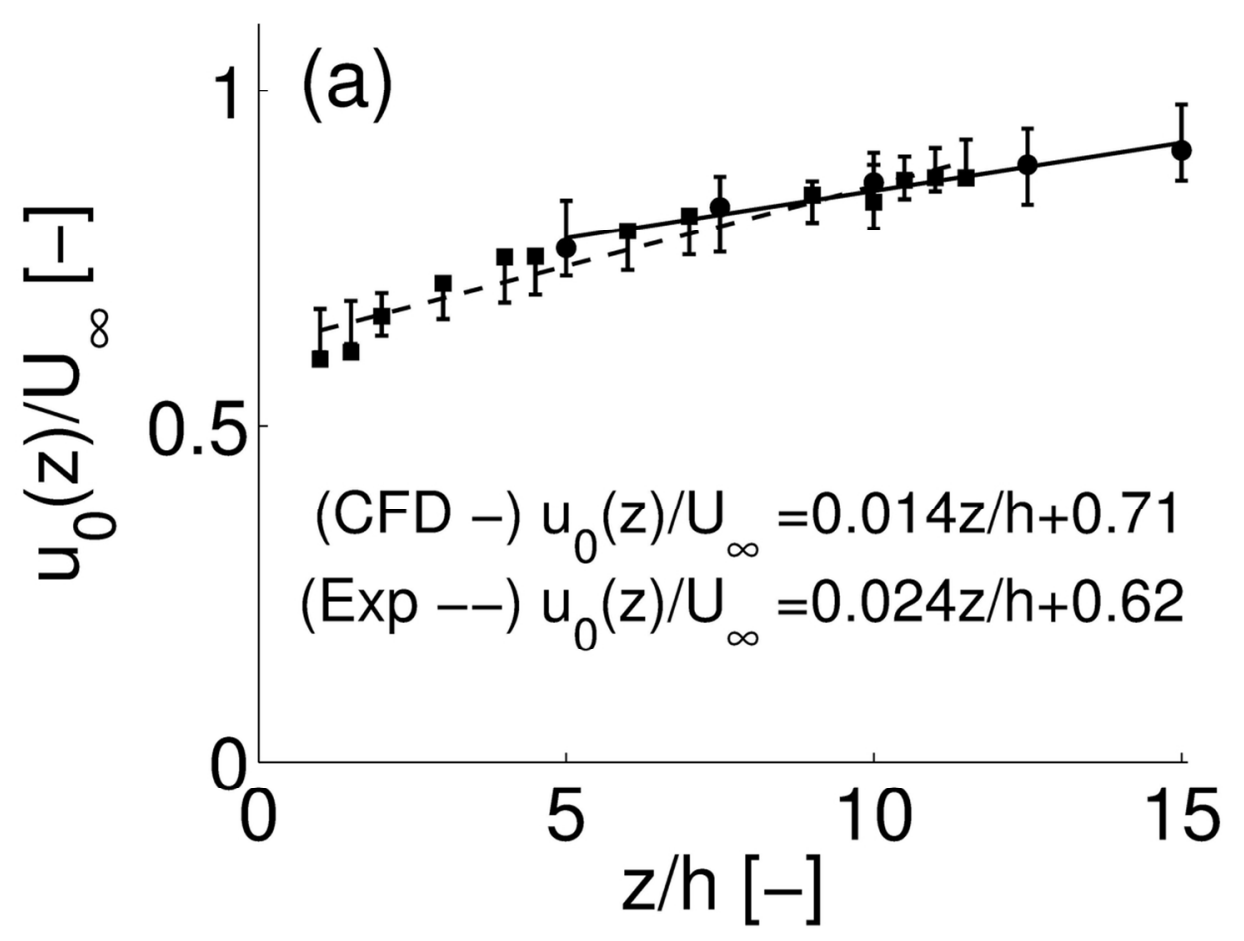

$111 \times 83 \mathrm{~mm}(300 \times 300 \mathrm{DPI})$ 


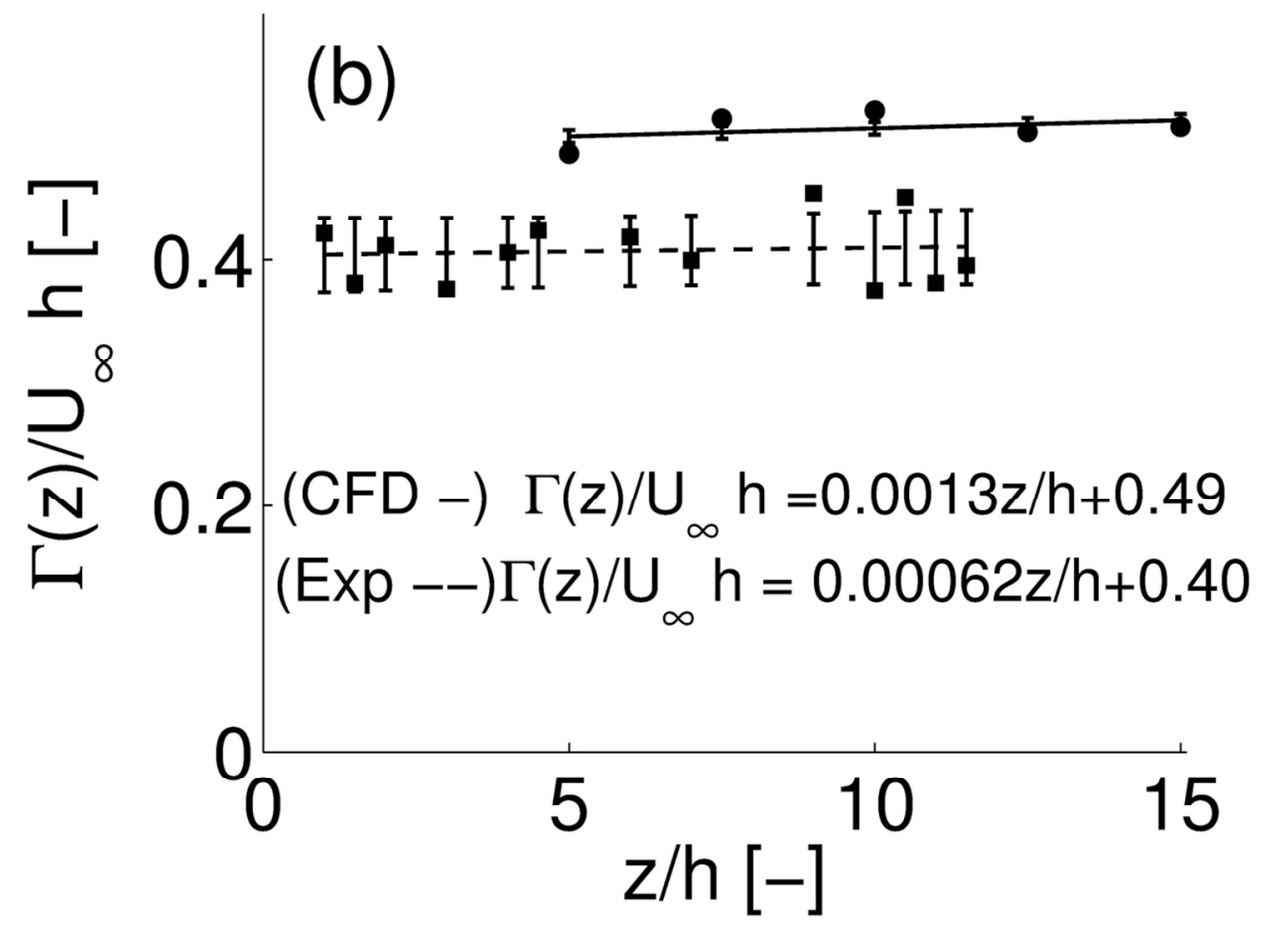

$111 \times 83 \mathrm{~mm}(300 \times 300 \mathrm{DPI})$ 


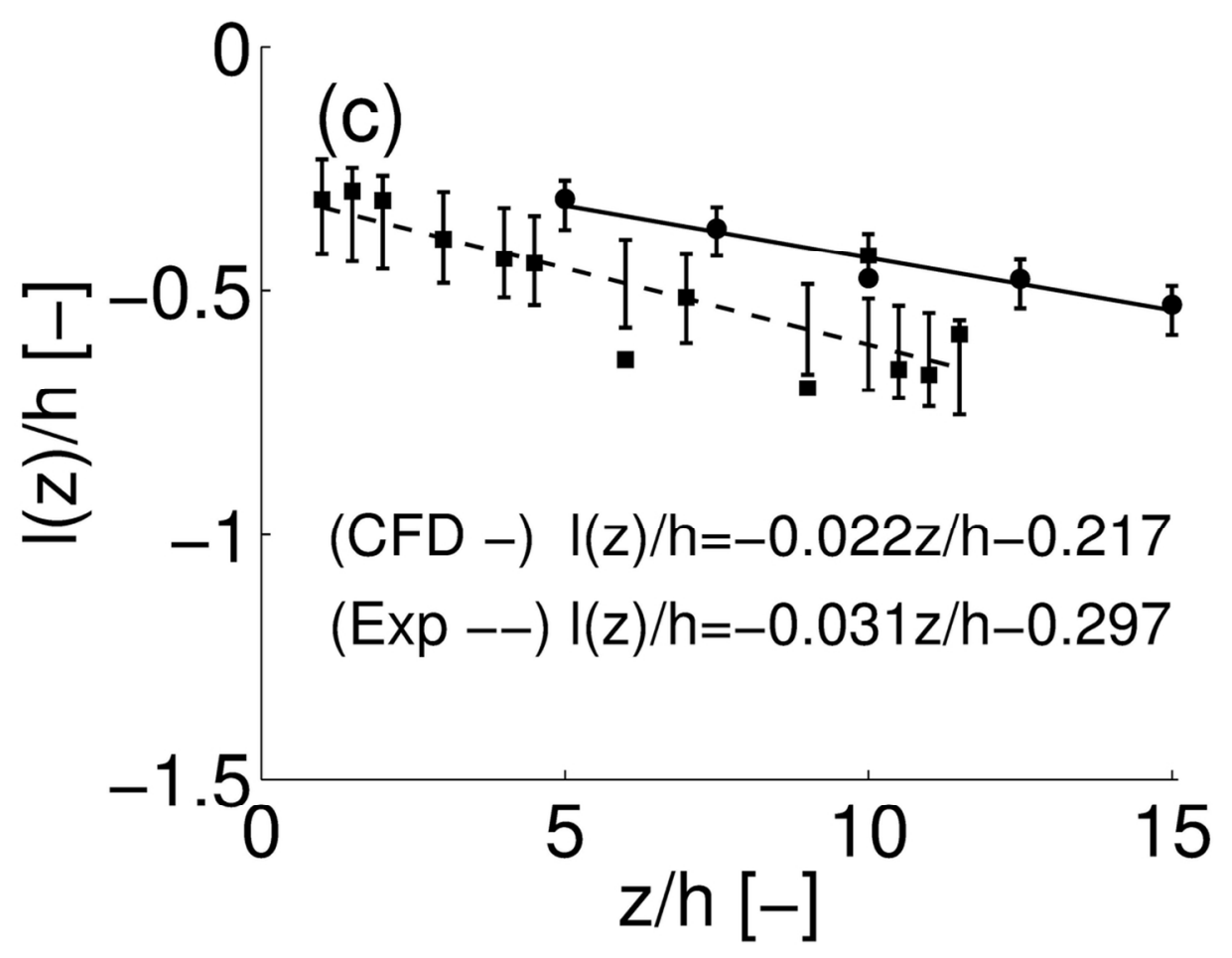

$111 \times 83 \mathrm{~mm}(300 \times 300$ DPI $)$ 


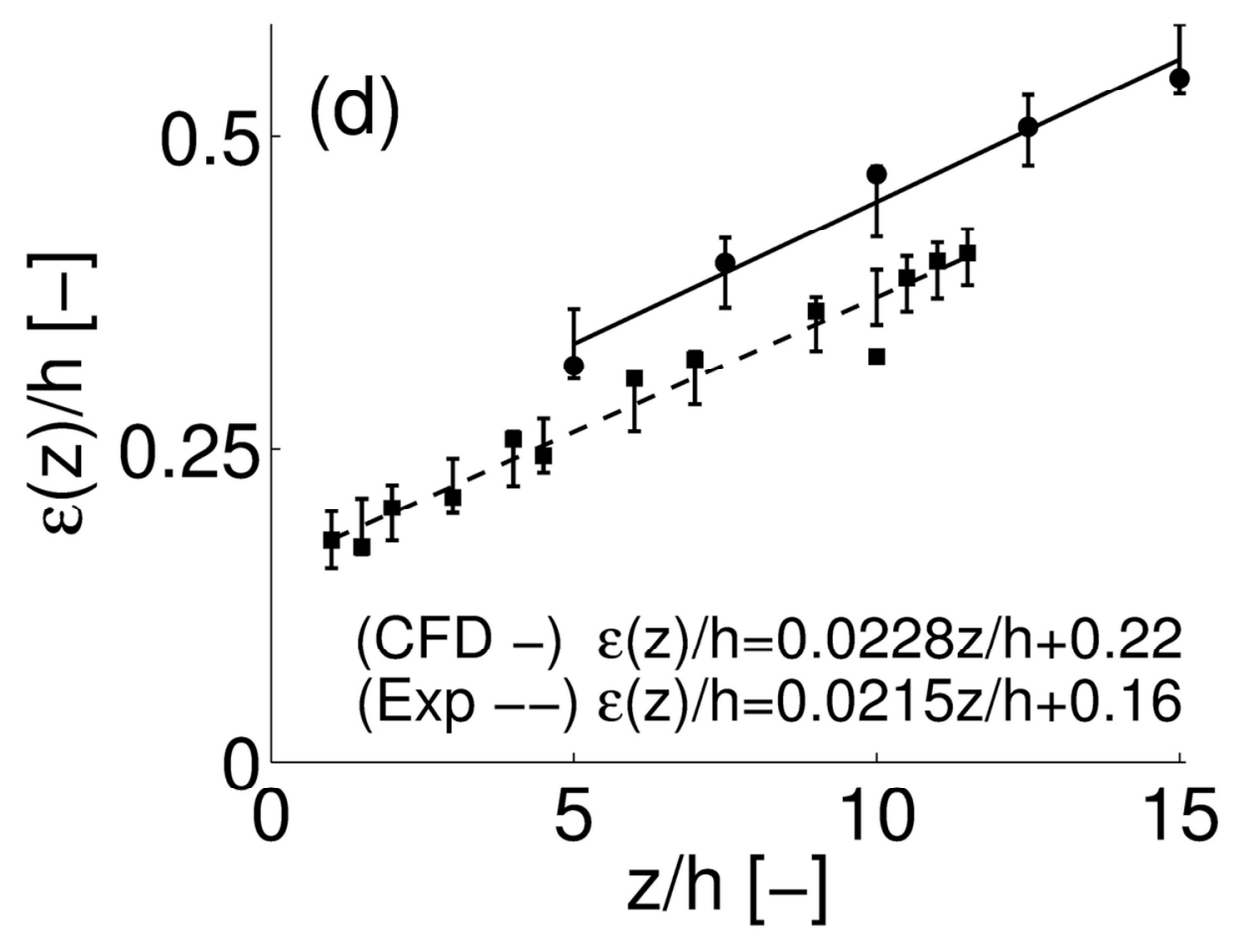

$111 \times 83 \mathrm{~mm}(300 \times 300 \mathrm{DPI})$ 


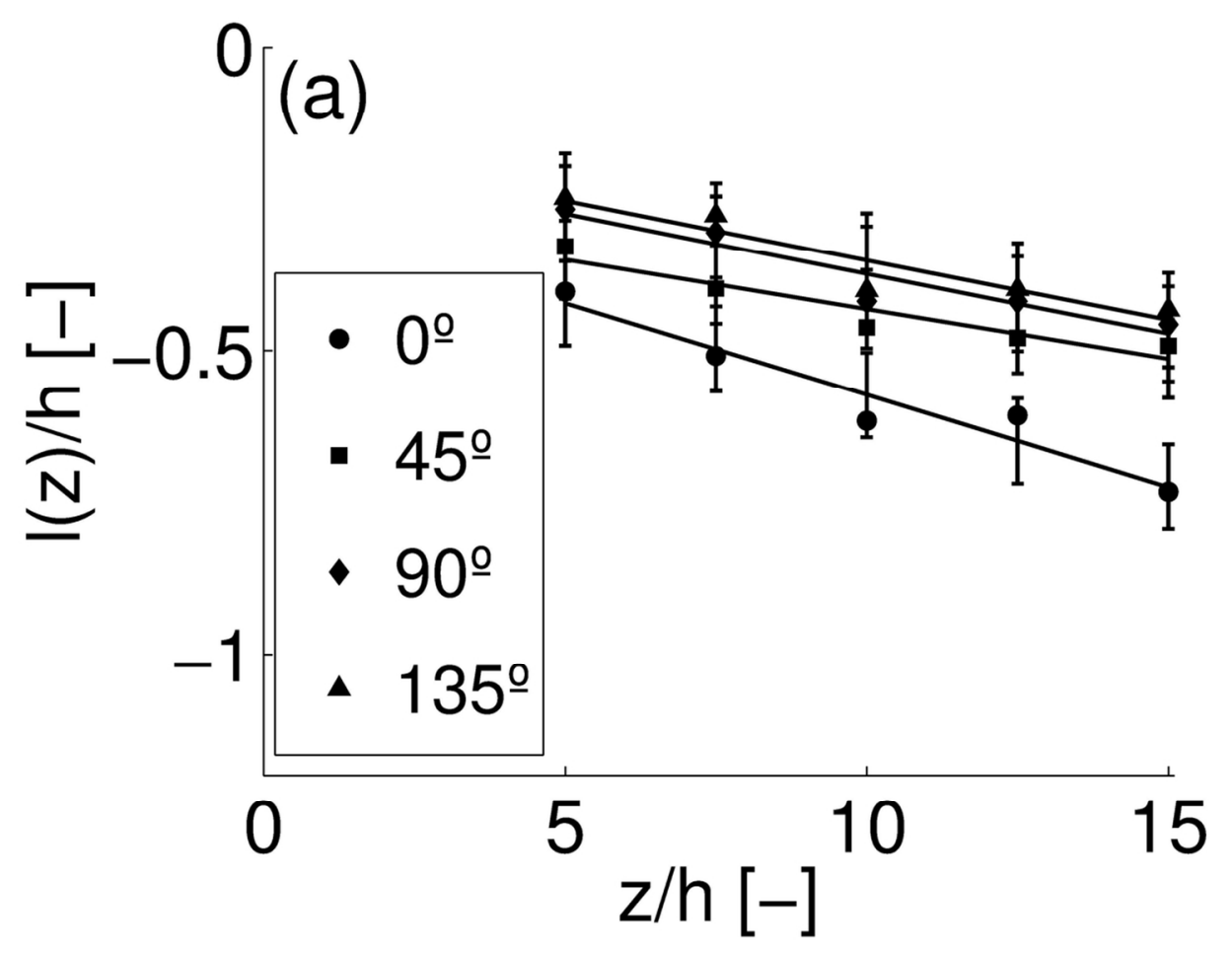

$111 \times 83 \mathrm{~mm}(300 \times 300$ DPI $)$ 


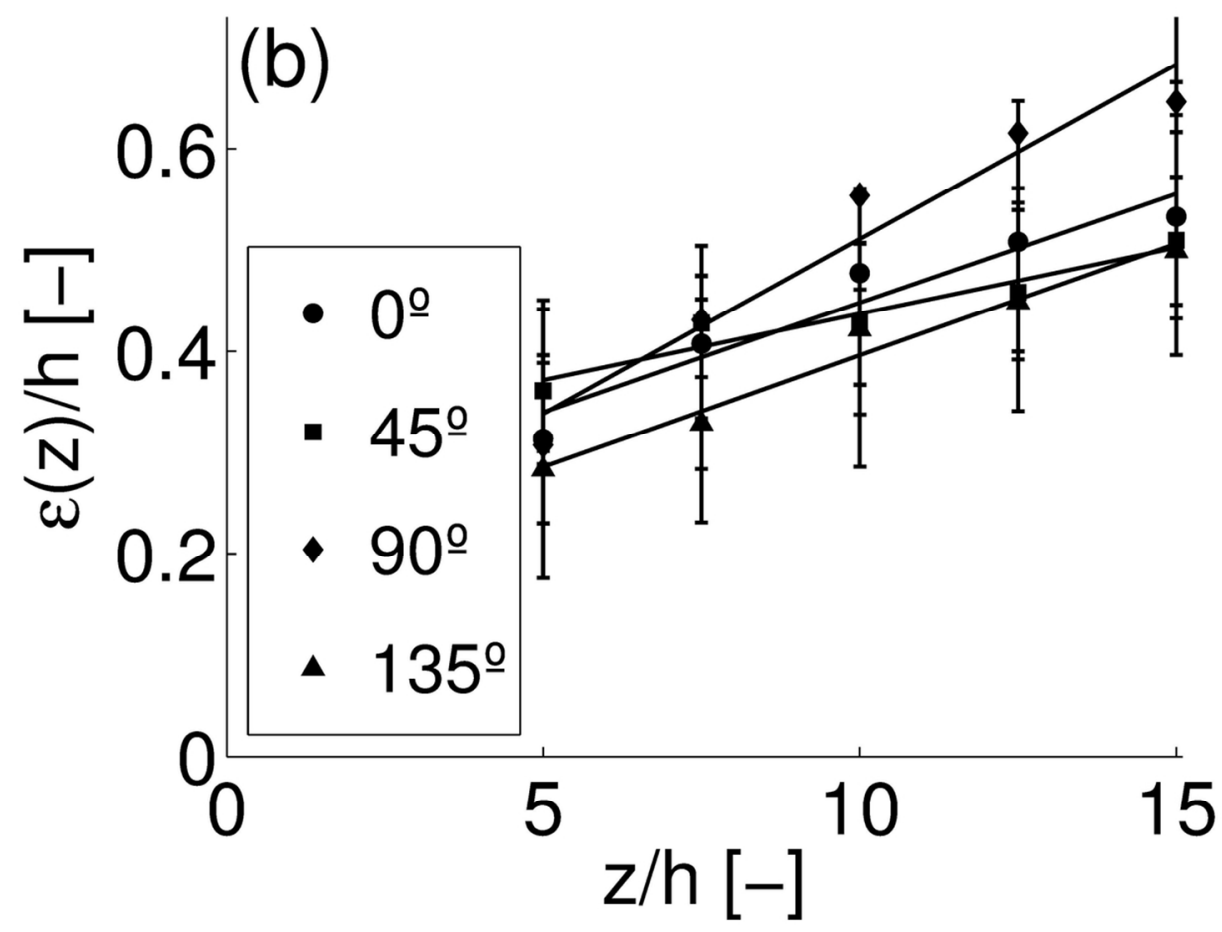

CFD results of the downstream evolution of the helical pitch (a) and the vortex radius (b) at different angles of $\$ \backslash$ Theta $\$: 0 \$ \wedge|\operatorname{circ} \$, 45 \$ \wedge| \operatorname{circ} \$, 90 \$ \wedge \mid \operatorname{circ} \$$ and $135 \$ \wedge \mid$ circ $\$$. $111 \times 83 \mathrm{~mm}$ (300 x 300 DPI) 


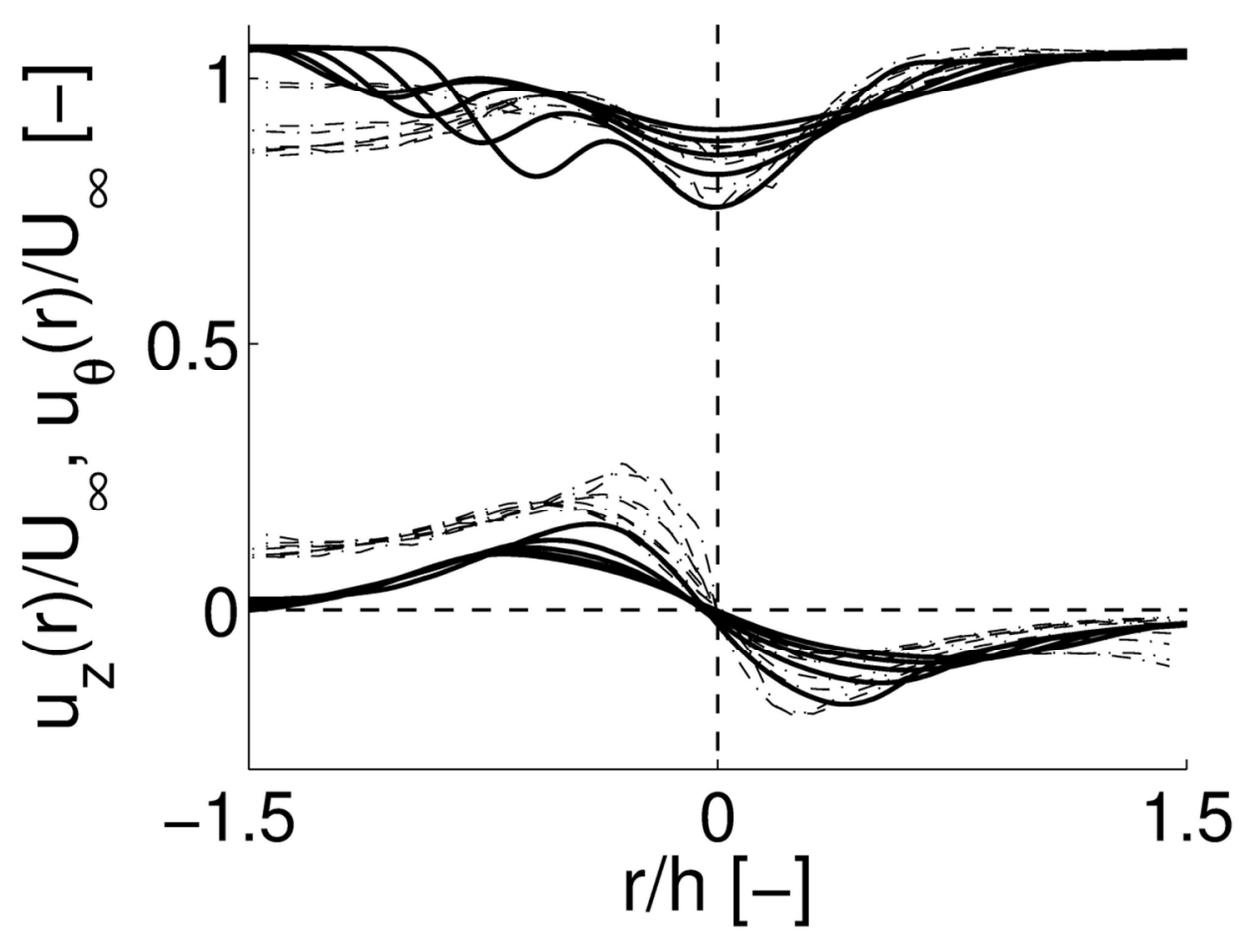

$111 \times 83 \mathrm{~mm}(300 \times 300$ DPI $)$ 


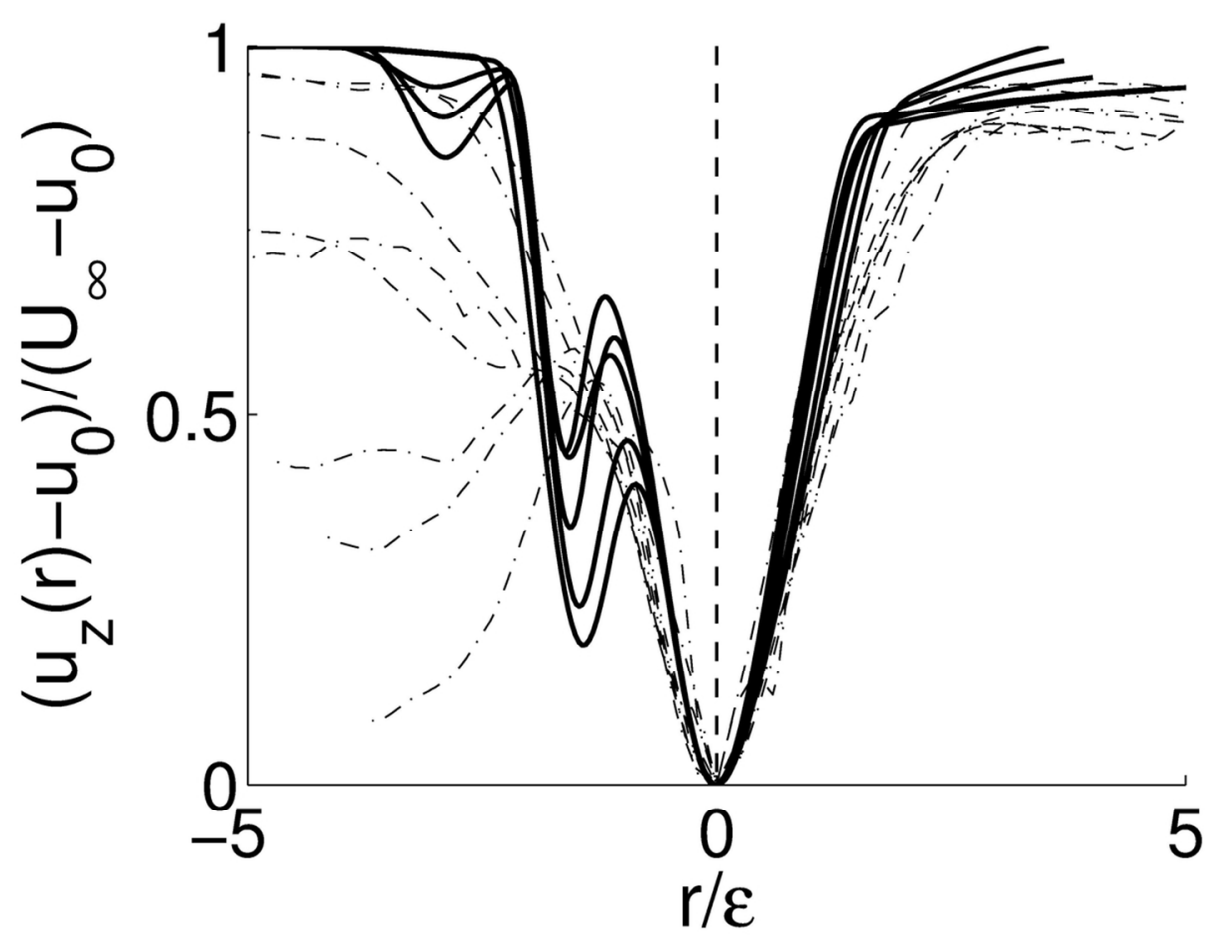

$111 \times 83 \mathrm{~mm}(300 \times 300$ DPI $)$ 


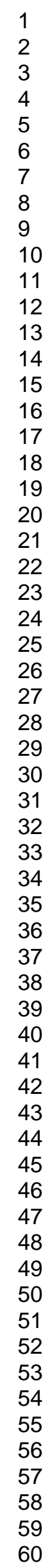

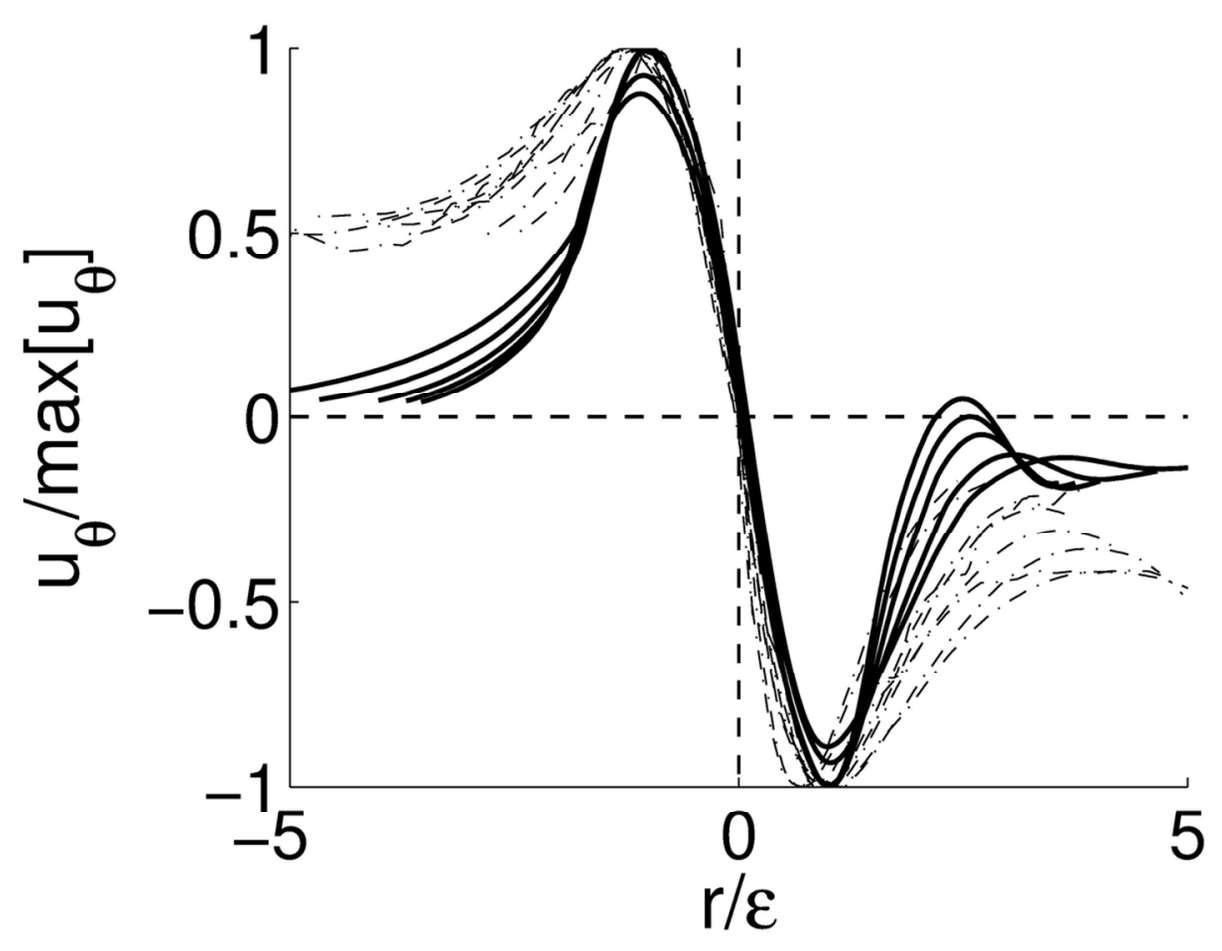

$111 \times 83 \mathrm{~mm}(300 \times 300$ DPI $)$ 


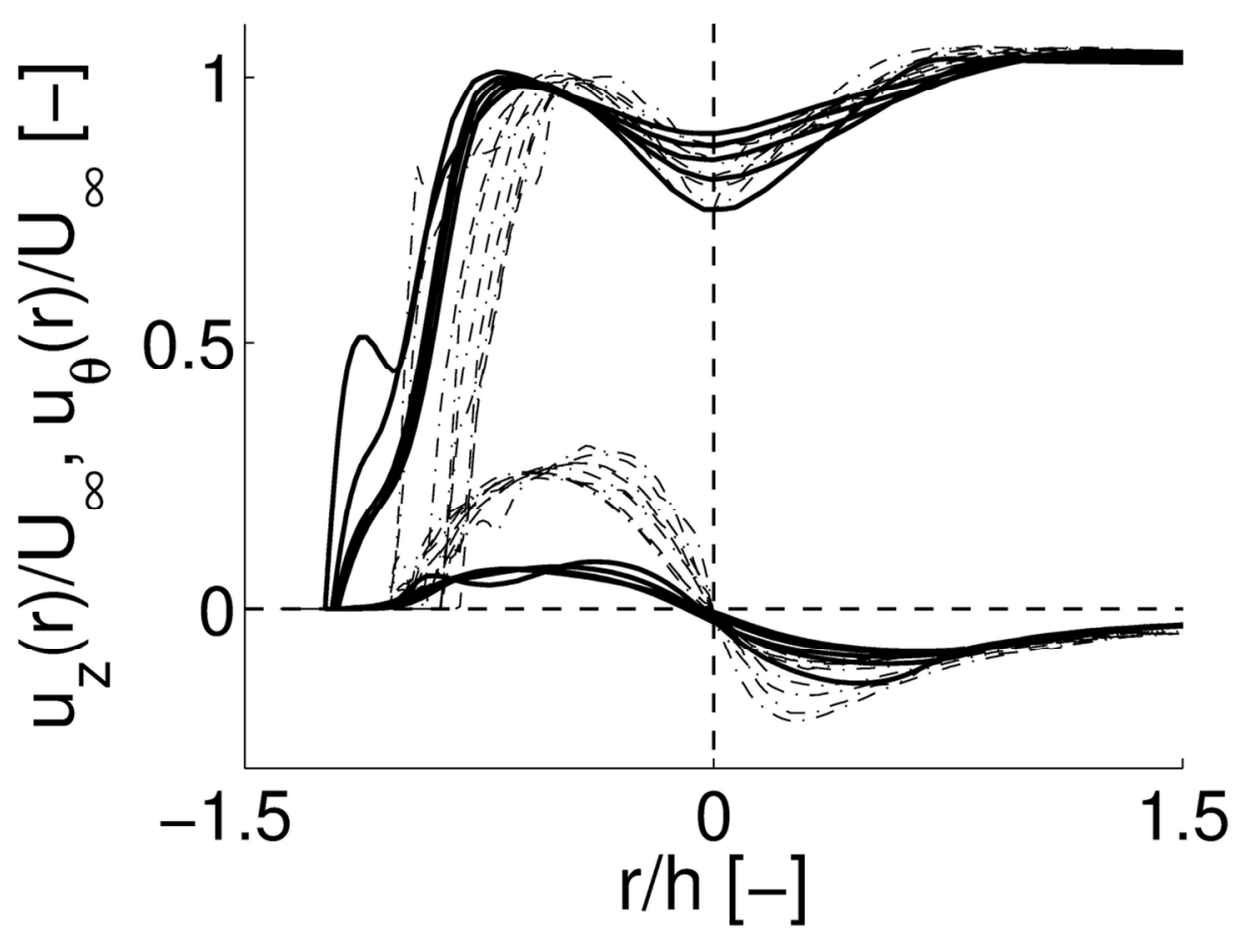

$111 \times 83 \mathrm{~mm}(300 \times 300$ DPI $)$ 


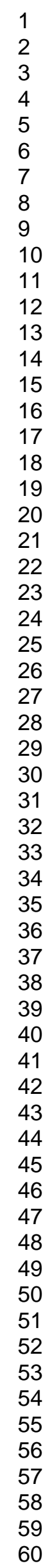

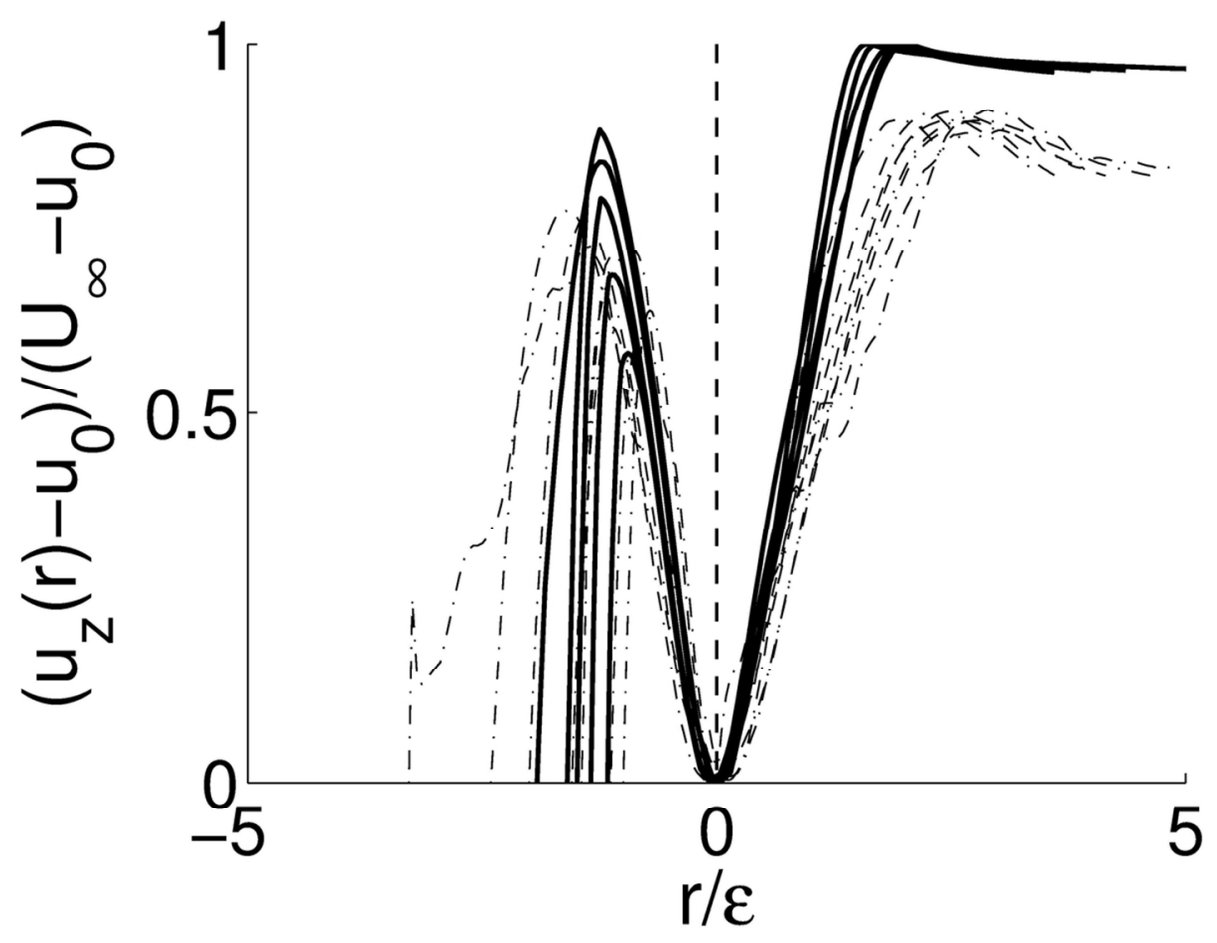

$111 \times 83 \mathrm{~mm}(300 \times 300$ DPI $)$ 


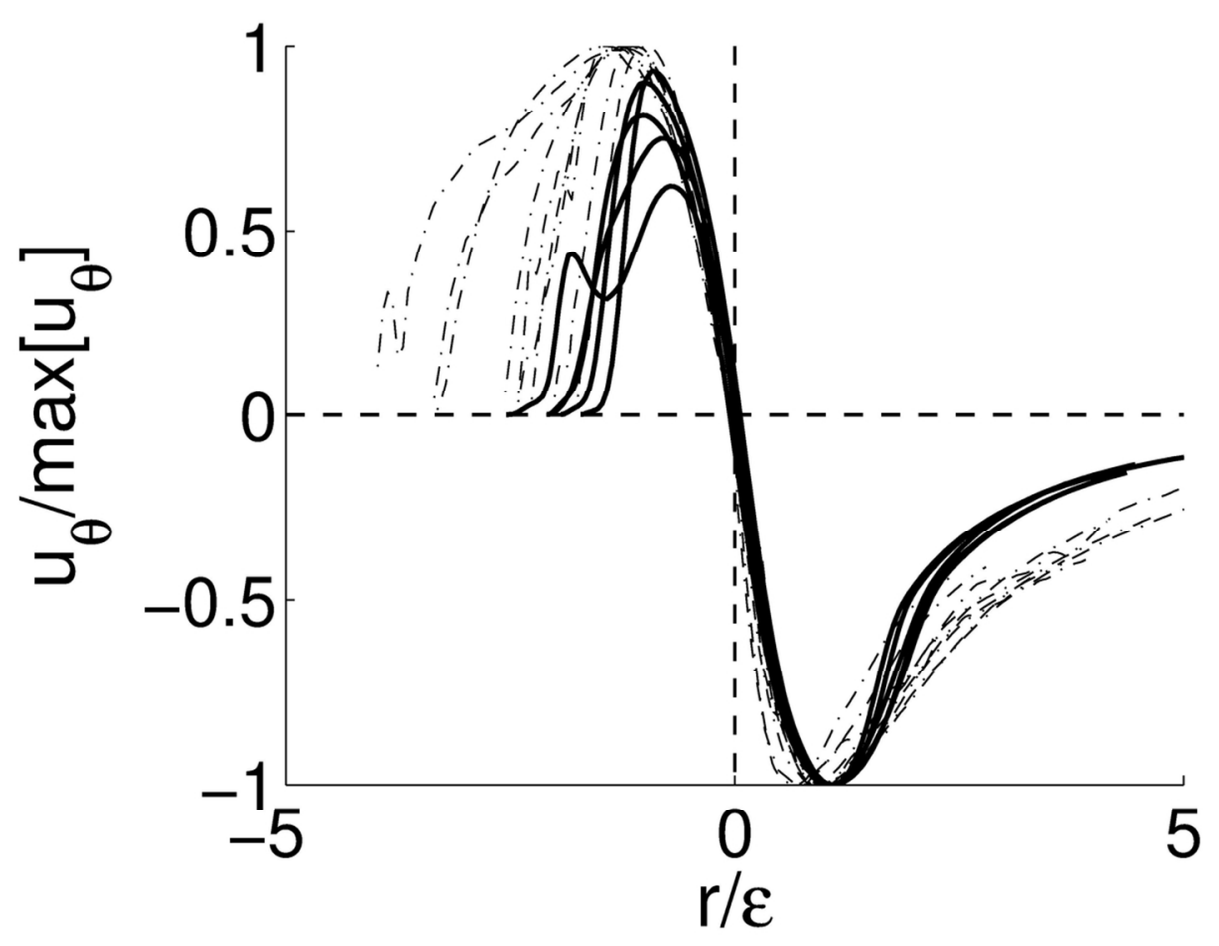

$111 \times 83 \mathrm{~mm}(300 \times 300$ DPI $)$

John Wiley \& Sons 


1
2
3
4
5
6
7
8
9
10
11
12
13
14
15
16
17
18
19
20
21
22
23
24
25
26
27
28
29
30
31
32
33
34
35
36
37
38
39
40
41
42
43
44
45
46
47
48
49
50
51
52
53
54
55
56
57
58
60

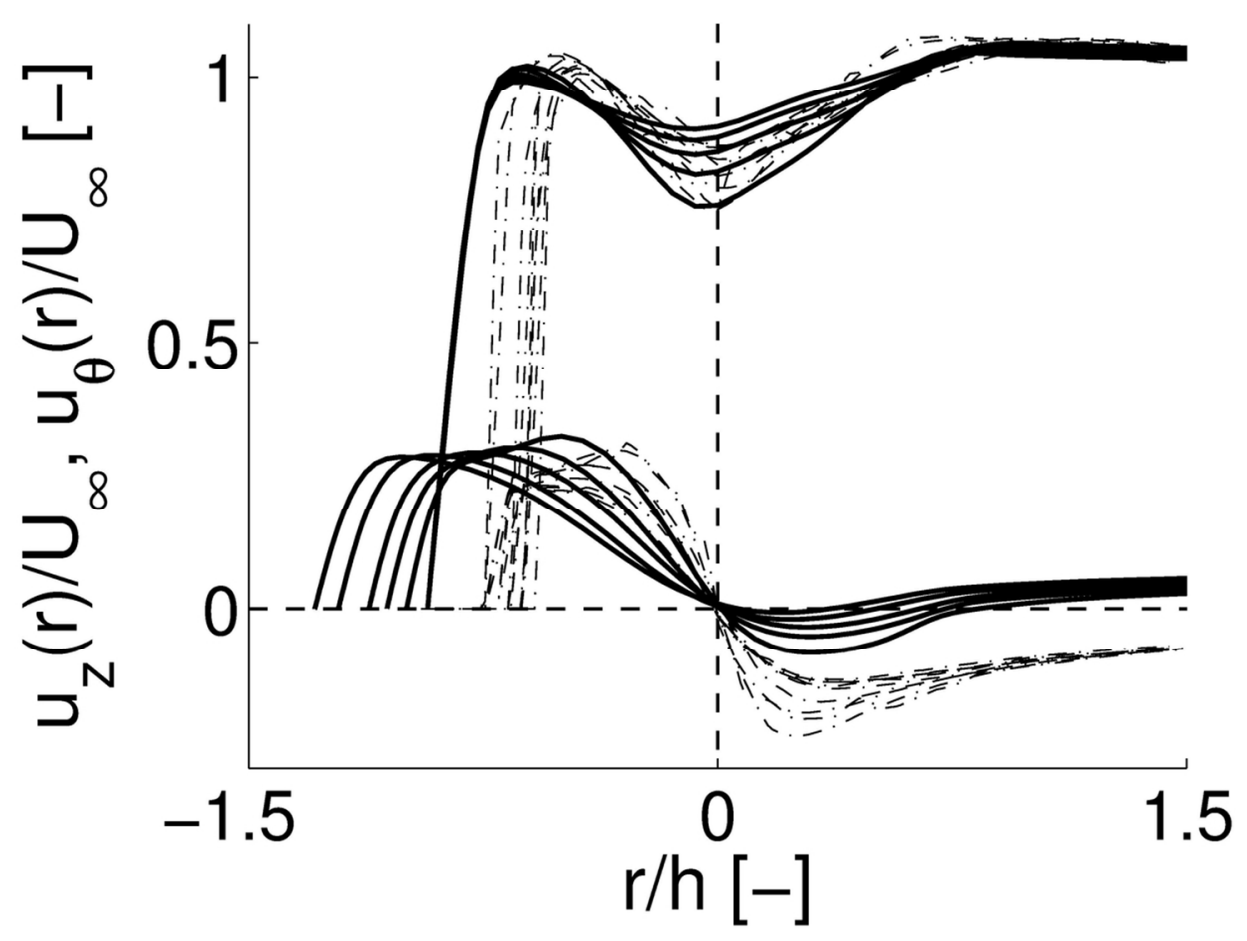

$111 \times 83 \mathrm{~mm}(300 \times 300$ DPI $)$ 


1
2
3
4
5
6
7
8
9
10
11
12
13
14
15
16
17
18
19
20
21
22
23
24
25
26
27
28
29
30
31
32
33
34
35
36
37
38
39
40
41
42
43
44
45
46
47
48
49
50
51
52
53
54
55
56
57
59
60

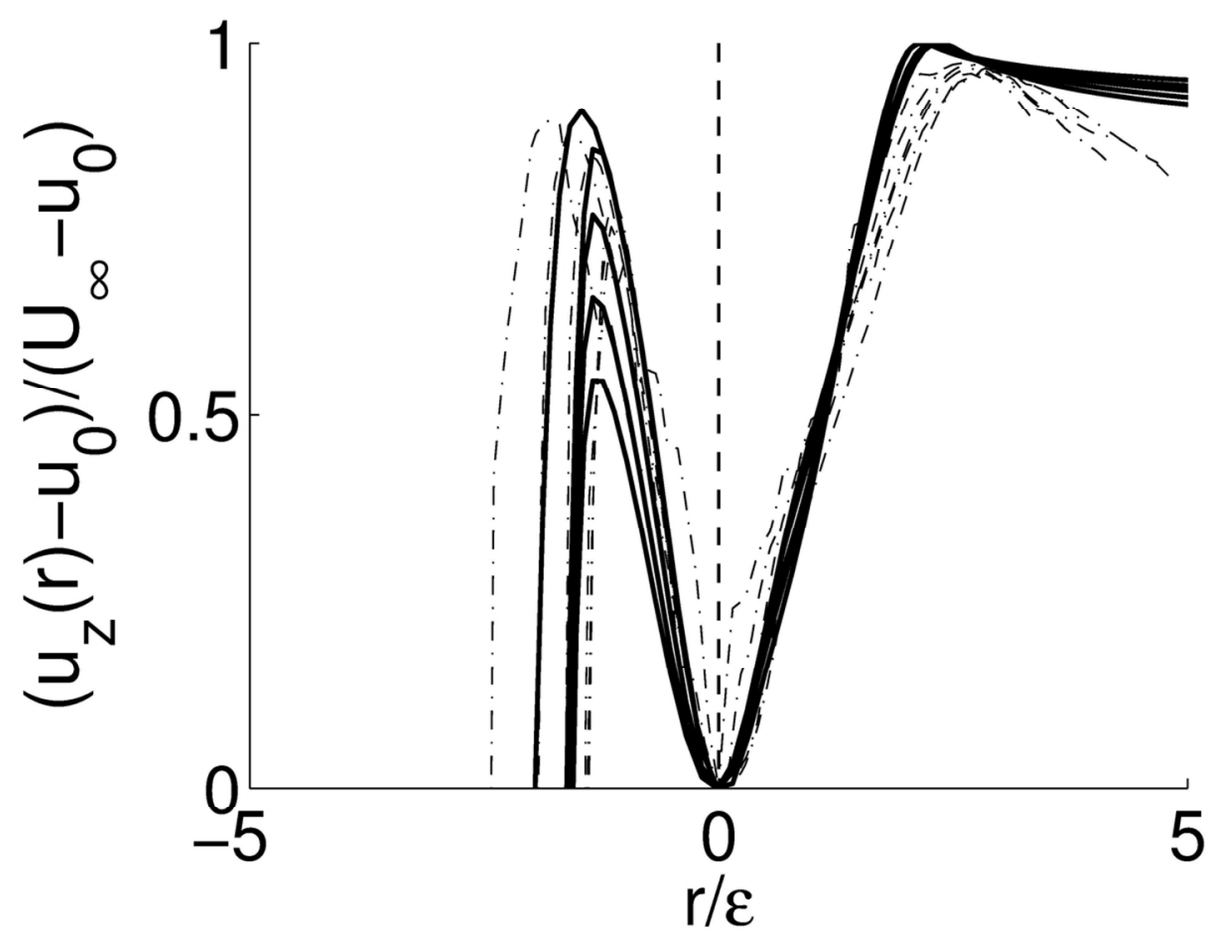

$111 \times 83 \mathrm{~mm}(300 \times 300$ DPI $)$ 


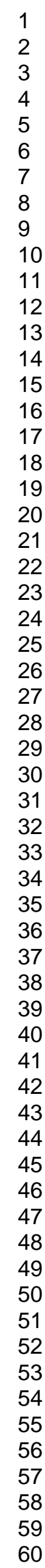

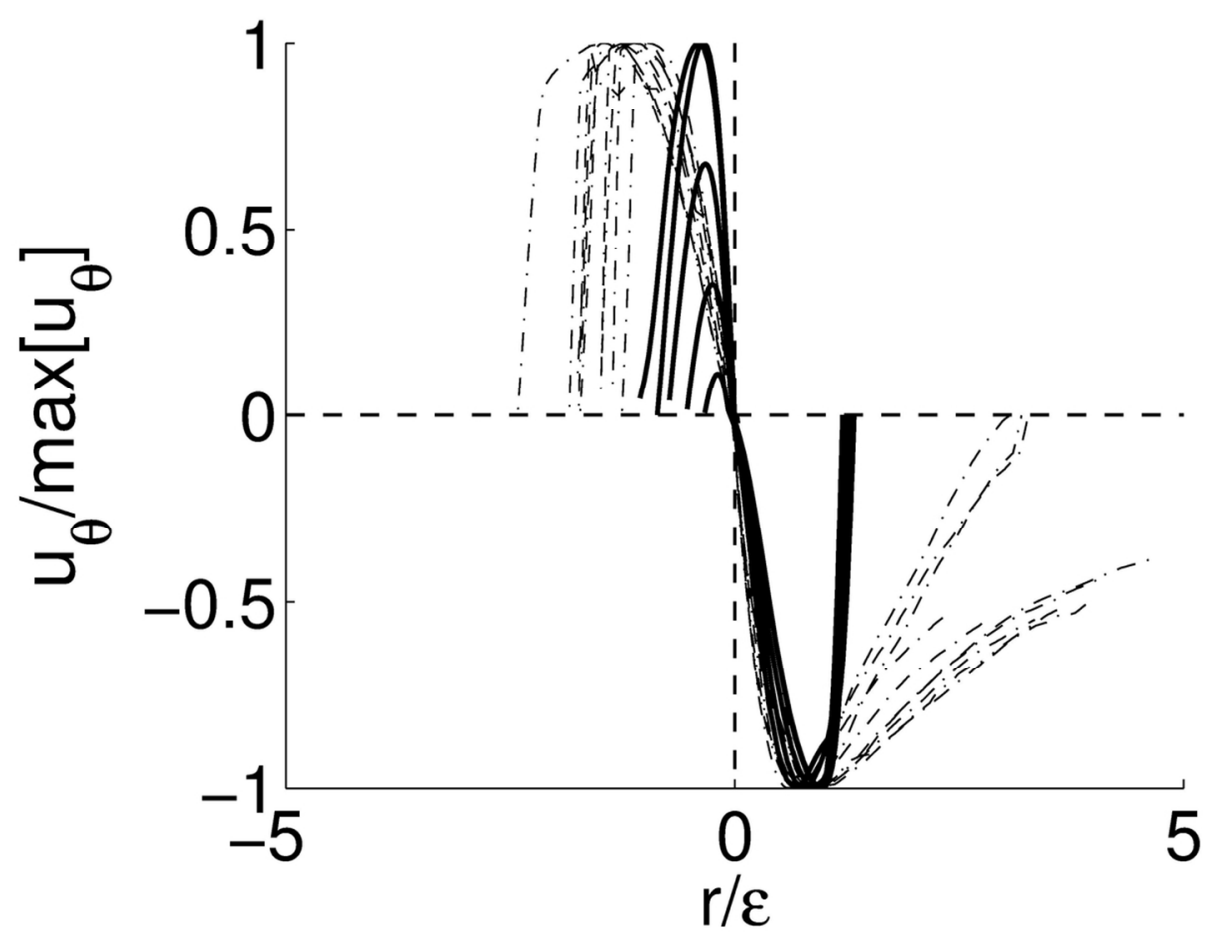

$111 \times 83 \mathrm{~mm}(300 \times 300 \mathrm{DPI})$ 


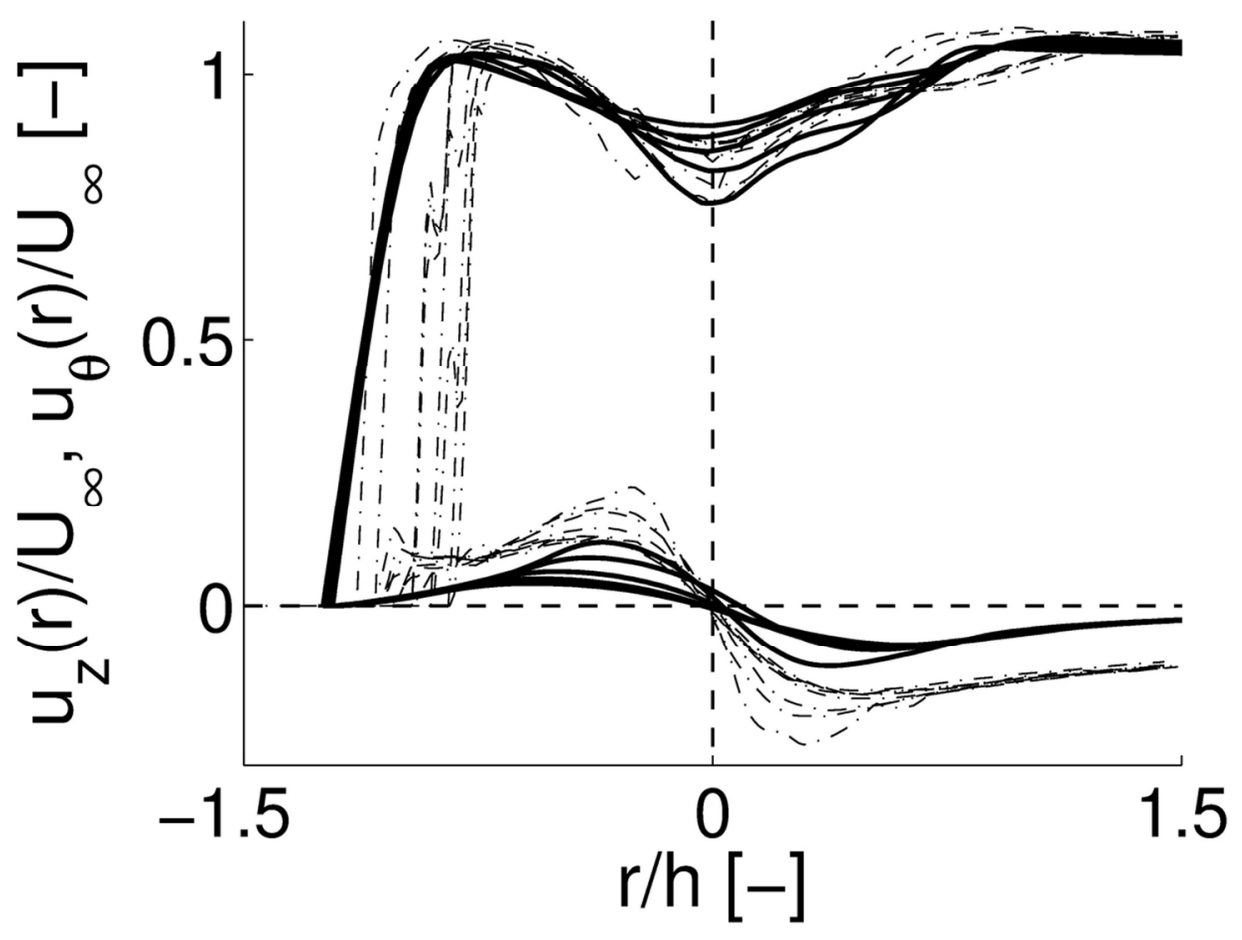

$111 \times 83 \mathrm{~mm}(300 \times 300$ DPI $)$ 


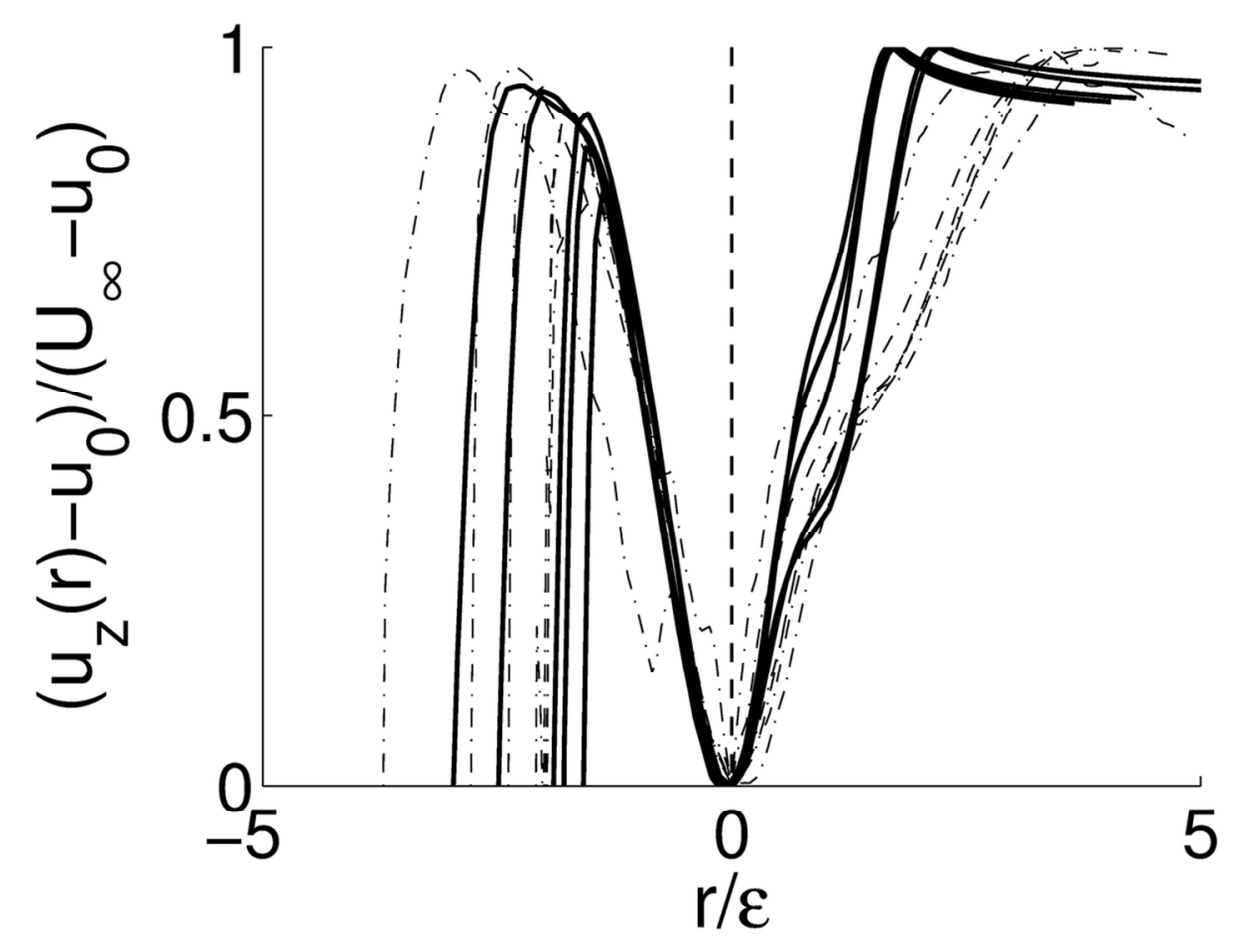

$111 \times 83 \mathrm{~mm}(300 \times 300$ DPI) 


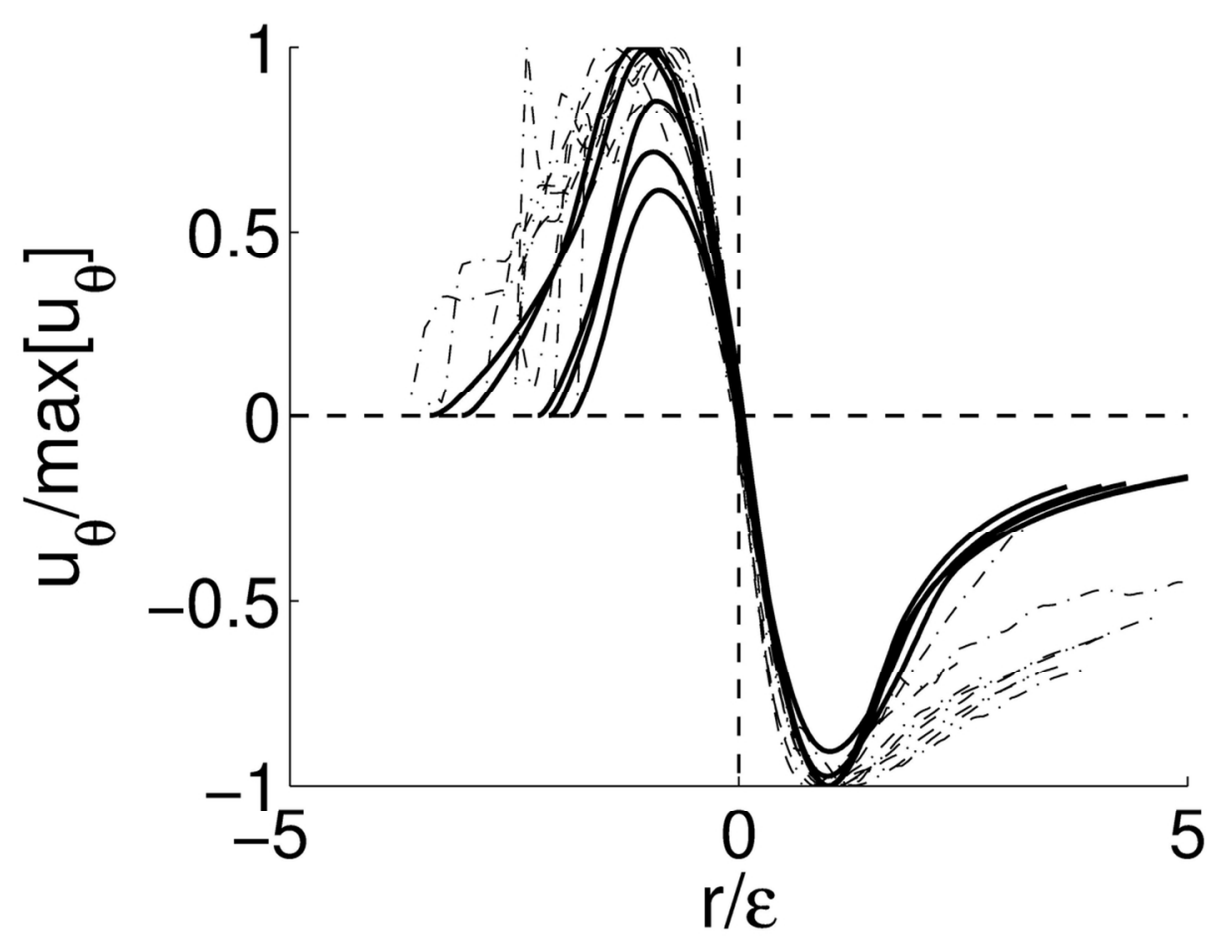

CFD (solid lines) and experimental (dash-dot lines) velocity profiles for various angles $\$ \backslash$ Theta $\$$ at different plane positions downstream of the VG. The left side shows the axial ( $\left.\$ u_{-} z \$\right)$ and azimuthal ( $\$ u_{-} \mid$theta $\$$ ) profiles and the middle and right sides show the axial and azimuthal profiles respectively, scaled by selfsimilarity variables. $111 \times 83 \mathrm{~mm}(300 \times 300$ DPI) 